\title{
Intrinsic excitability mechanisms of neuronal ensemble formation
}

\author{
Tzitzitlini Alejandre-García, Samuel Kim, Jesús Pérez-Ortega and Rafael Yuste \\ Neurotechnology Center, Dept. Biological Sciences, Columbia University, New York, NY 10027, USA
}

Running Head: Mechanisms of neuronal ensembles

Correspondence to:

Tzitzitlini Alejandre-García

902 NWC Building 550 West 120 Street, Box 4822, New York, NY, 10027.

Email: at3415@columbia.edu

Conflict of Interest: The authors declare no competing financial interests

Acknowledgments: We thank James Holland, for his assistance and members of the Yuste Lab for useful comments. Supported by R01EY011787 and R01MH115900. R.Y. is an Ikerbasque Research Professor at the Donostia International Physics Center (DIPC). The authors have no competing financial interests to declare. S.K. and R.Y. conceived the project. S.K. and T.A. performed experiments and T.A. and R.Y. wrote the paper. T.A., S.K. and J.P. analyzed the data. All authors planned experiments, discussed results and edited the paper. R.Y. assembled and 7 directed the team and secured funding and resources. 


\begin{abstract}
9 Neuronal ensembles are coactive groups of cortical neurons, found in both spontaneous and 10 evoked activity, which can mediate perception and behavior (Cossart et al., 2003; Buzsáki, 2010;

11 Carrillo-Reid et al., 2019; Marshel et al., 2019). To understand the mechanism that lead to the

12 formation of neuronal ensembles, we generated optogenetically artificial photo-ensembles in

13 layer 2/3 pyramidal neurons in brain slices of mouse visual cortex from both sexes, replicating an

14 optogenetic protocol to generate ensembles in vivo by simultaneous coactivation of neurons

15 (Carrillo-Reid et al. 2016). Using whole-cell voltage-clamp recordings from individual neurons and

16 connected pairs, we find that synaptic properties of photostimulated were surprisingly unaffected,

17 without any signs of Hebbian plasticity. However, extracellular recordings revealed that

18 photostimulation induced strong increases in spontaneous action potential activity. Using

19 perforated patch clamp recordings, we find increases in neuronal excitability, accompanied by

20 increases in membrane resistance and a reduction in spike threshold. We conclude that the

21 formation of neuronal ensemble by photostimulation is mediated by cell-intrinsic changes in

22 excitability, rather than by Hebbian synaptic plasticity or changes in local synaptic connectivity.

23 We propose an "iceberg" model, by which increased neuronal excitability makes subthreshold

24 connections become suprathreshold, increasing the functional effect of already existing synapses

25 and generating a new neuronal ensemble.
\end{abstract}


Alejandre-García et al., p.3

27 The function of brain is anchored on the activity of groups of connected neurons, forming 28 microcircuits (Schüz and Braitenberg, 2001; Shepherd, 2004). These groups of coactive neurons, 29 or ensembles (also known as assemblies or attractors), exhibit synchronous or correlated activity 30 (Lorente De Nó, 1938; Hebb, 1949; Hopfield, 1982; Abeles, 1991; Cossart et al., 2003; Buzsáki, 31 2010; Miller et al., 2014; Yuste, 2015; Carrillo-Reid et al., 2016, 2019; Marshel et al., 2019), and 32 appear critical for brain function (Hoshiba et al., 2017) and memory formation (Hebb, 1949). In 33 the cerebral cortex, these states may arise from patterns of multineural activity that persist 34 spontaneously after an initial stimulus, generating recurrent activity (reverberations) (Lorente de 35 Nó, 1933; Churchland and Sejnowski, 1992). Cortical ensembles correlate with different functions 36 of the animal, not just memory storage, but also representation of thoughts, and percepts as 37 demonstrated in the primary visual cortex of awake mice during spontaneous and visually evoked 38 activity patterns in neurons (Miller et al., 2014a; Carrillo-Reid et al., 2019; Marshel et al., 2019).

39 Moreover, the disorganization of neuronal ensembles is associated with neuropsychiatric 40 disorders such as schizophrenia (Hamm et al., 2017).

41 One hypothesis of how an ensemble could be formed was first proposed by Donald Hebb in 1949.

42 According to this rule, the persistent and repeated activation of connected neurons induce 43 metabolic changes or growth of processes in one or both cells that strengthens those connections.

44 One mechanism that fulfills Hebb's rule is Long-Term Synaptic Potentiation (LTP), originally 45 defined as a long-lasting increase in synaptic efficacy after a high frequency burst electrical 46 stimulation (Bliss and Gardner-Medwin, 1973). Accordingly, changes in synaptic weights 47 produces preferential connectivity that controls the flow of activity within and between neuronal 48 ensembles (Hoshiba et al., 2017). Since its initial discovery (Bliss and Gardner-Medwin, 1973), 49 LTP has been widely established as the cellular correlate for memory and learning. Indeed, recent 
50 studies have supported the idea that Hebbian plasticity could underlie ensemble formation in vivo

51 (Carrillo-Reid et al., 2016), since the two-photon optogenetic synchronous stimulation of a groups

52 of neurons 50 to 100 times bound them together into an imprinted ensemble which become

53 spontaneously coactive. Therefore, co-activation of a group of neurons in awake mice can create

54 a new stable artificial ensemble (Carrillo-Reid et al., 2016). This result is consistent with the

55 Hebbian hypothesis on the formation of neuronal ensembles, but no direct evidence has yet been

56 presented to demonstrate that the formation of ensembles depends on changes in synaptic

57 connections in neuronal circuit.

As an alternative hypothesis, experimental evidence has revealed widespread activity-dependent cell-intrinsic mechanisms in cortical circuits (Ryan et al., 2015; Titley et al., 2017). This suggests that the formation and stability of an ensemble could be carried out by cell-autonomous intrinsic mechanisms (Abraham et al., 2019). This hypothesis is consistent with experiments where the

62 activation of specific patterns of cellular activity in hippocampus recalls stored memories, even

63 under treatment with protein synthesis inhibitor which should prevent LTP (Ryan et al., 2015;

64 Tonegawa et al., 2015). Moreover, Purkinje cells can acquire and represent a specific input with

65 a temporal pattern of activity, suggesting that patterns of activity may be imprinted intrinsically by

66 the neurons itself (Johansson et al., 2014). Thus, changes excitability state of the neurons may

67 contribute to ensemble consolidation, rather than the reinforcement of its synapses, as Hebb

68 proposed (Disterhoft and Oh, 2006; Pignatelli et al., 2019).

69 In order to test these alternative mechanisms of ensemble formation. we built photo-ensembles

70 in mouse neocortical slices, following an in vivo optogenetic protocol (Carrillo-Reid et al., 2016).

71 We then directly measured electrophysiological properties of photostimulated neurons, finding a

72 lack of changes of synaptic properties yet generalized increases in cell-intrinsic excitability. We

73 conclude that ensemble formation is a non-Hebbian phenomenon. 
75 All procedures were performed in accordance with the U.S. National Institutes of Health and

76 Columbia University Institutional Animal Care and Use Committee guidelines. Experiments were

77 carried out on C57BL/6 transgenic mice (Vglut1-Cre, Jackson Laboratories;

78 RRID:IMSR_JAX:00064) of both sexes at postnatal day of 1 - 2 months. Animals were housed on

79 a $12 \mathrm{~h}$ light-dark cycle with food and water ad libitum.

80 Viral injection, after 1 month postnatal, animals were anesthetized with $2 \%$ isoflurane on a

81 headfiexed stereotactic apparatus. After sterilizing the incision site, the skin was opened and,

82 using an FG 1/4 dental drill, a small hole goes thin in the skull over visual primary cortex (2.5 mm

83 lateral and $0.3 \mathrm{~mm}$ anterior from the lambda, $200 \mu \mathrm{m}$ from pia). We injected $300 \mathrm{~nL}$ of virus at a

84 rate of 30-40 nL/s, using a microsyringe pump (Micro 4), a Hamilton 7653-01 and a glass pipette.

85 Once pipette was placed, we waited $5 \mathrm{~min}$ before and after virus injection and finally closing the

86 scalp with sutures. Viruses injected were: Cre-dependent AAV-CAG-DIO-ChroME-P2A-H2B-

87 mRuby3 (Addgene viral prep \# 108912-AAV9) or AAVDJ-CaMKIla-C1V1(E162T)-TS-p2A-EYFP-

88 WPRE (Stanford University Gene Vector and Virus Core). Mice were used for electrophysiology

89 experiments $2-3$ weeks postinjection.

90 Brain slices, mice were anesthetized with ketamine/xylazine. After transcardiacal perfusion

91 procedure and posterior cervical dislocation, brain sagittal slices were obtained as described (Ting

92 et al., 2018). The brains were quickly dissected and cooled in a continuously gassed $\left(95 \% \mathrm{O}_{2}\right.$ and

$\left.935 \% \mathrm{CO}_{2}\right)$ icy NMDG-HEPES artificial cerebrospinal fluid (aCSF). Transcardiacal perfusion and

94 cutting solution, containing (in mM): $92 \mathrm{NMDG}, 2.5 \mathrm{KCl}, 1.25 \mathrm{NaH}_{2} \mathrm{PO}_{4}, 30 \mathrm{NaHCO}_{3}, 20 \mathrm{HEPES \text {, }}$

9525 glucose, 2 thiourea, $5 \mathrm{Na}$-ascorbate, $3 \mathrm{Na}$-pyruvate, $0.5 \mathrm{CaCl}_{2}$, and $10 \mathrm{MgSO}_{4}$ with pH titrated

96 to 7.3-7.4 with $\mathrm{HCl}$, or 222 Sucrose, $2.6 \mathrm{KCl}, 27 \mathrm{NaHCO}_{3}, 1.5 \mathrm{NaH}_{2} \mathrm{PO} 4,2 \mathrm{MgSO}_{4}$, and $2 \mathrm{CaCl}_{2}$.

$97300 \mu \mathrm{m}$ thick sagittal slices were cut on a Leica VT1200 S vibratome (Leica Biosystems) and 
98 allowed to recover for 20 min at $34^{\circ} \mathrm{C}$ HEPES holding aCSF solution (in $\mathrm{mM}$ ): $92 \mathrm{NaCl}, 2.5 \mathrm{KCl}$,

$991.25 \mathrm{NaH}_{2} \mathrm{PO}_{4}, 30 \mathrm{NaHCO}_{3}, 20$ HEPES, 25 glucose, 2 thiourea, 5 Na-ascorbate, 3 Na-pyruvate,

$1002 \mathrm{CaCl}_{2}$, and $2 \mathrm{MgSO}_{4}$. Titrate $\mathrm{pH}$ to 7.3-7.4 with $\mathrm{NaOH}$. Finally, slices were transferred to aCSF

101 containing the following (in $\mathrm{mM}$ ): $124 \mathrm{NaCl}, 2.5 \mathrm{KCl}, 1.25 \mathrm{NaH} 2 \mathrm{PO} 4,24 \mathrm{NaHCO}, 12.5$ glucose,

$1025 \mathrm{HEPES}, 2 \mathrm{CaCl}_{2} \cdot 2 \mathrm{H} 2 \mathrm{O}$, and $2 \mathrm{MgSO} 4 \cdot 7 \mathrm{H}_{2} \mathrm{O}$ with pH titrated to $7.3-7.4$, or $126 \mathrm{NaCl}, 3 \mathrm{KCl}$,

$1031.145 \mathrm{NaH}_{2} \mathrm{PO}_{4}, 26 \mathrm{NaHCO}_{3}, 10$ Glucose, $2 \mathrm{MgSO}_{4}$, and $2 \mathrm{CaCl}_{2}$. Slices rested in dark for at least

$1041 \mathrm{hr}$ before use.

105 Patch Clamp Recordings. Brain slices were carefully placed in the recording chamber on an 106 upright microscope (Olympus, $\mathrm{BX} 50 \mathrm{WI})$, they were continuously perfused with gassed $\left(95 \% \mathrm{O}_{2}\right.$

107 and $5 \% \mathrm{CO}_{2}$ ) aCSF at $5 \mathrm{ml} / \mathrm{min}$. All electrophysiological recordings were performed using patch

108 pipettes, made from borosilicate glass (World Precision Instruments), pulled with a micropipette 109 puller (DMZ-Universal puller), in order to get $4-6 \mathrm{M} \Omega$ as final resistances.

110 For whole-cell, patch pipettes were filled with intracellular solution containing the following (in $111 \mathrm{mM}$ ): $130 \mathrm{~K}$-gluconate, $20 \mathrm{KCl}, 10 \mathrm{HEPES}, 10 \mathrm{Na}_{2}$-phosphocreatine, 4 Mg-ATP, 0.03 Na $\mathrm{NaTP}_{\text {, }}$ 112 and titrated to $\mathrm{pH} 7.3$ with $\mathrm{KOH}$, or $135 \mathrm{~K}-\mathrm{MeSO}_{4}, 5 \mathrm{KCl}, 5 \mathrm{HEPES}, 0.5 \mathrm{EGTA}, 10 \mathrm{Na}_{2^{-}}$ 113 phosphocreatine, $2 \mathrm{Mg}-\mathrm{ATP}, 0.5 \mathrm{Na}_{2}-\mathrm{GTP}$, and titrated to $\mathrm{pH} 7.3$ with $\mathrm{KOH}$. For cell-attached, 114 pipettes were filled with aCSF solution. For perforated patch clamp, pipette solution containing: $115130 \mathrm{~K}$-gluconate, $20 \mathrm{KCl}, 10 \mathrm{HEPES}, 2 \mathrm{MgCl}_{2}, 4 \mathrm{Mg}-\mathrm{ATP}, 0.03 \mathrm{Na}_{2}$-GTP, and titrated to $\mathrm{pH} 7.3$ 116 with $\mathrm{KOH} .50-100 \mathrm{ng} / \mathrm{ml}$ gramicidin (sigma Aldrich) dissolved in dimethyl sulfoxide (DMSO). To 117 allow membrane sealing, the tip was immersed in clean pipette solution and then the pipette was 118 back-filled with gramicidin containing solution.

119 In each case, the patch pipette was placed in contact with the cell with the aid of a SM-5 120 micromanipulator (Luigs \& Neumann). Stimulation and data acquisition were sampled at $10 \mathrm{kHz}$ 121 and low pass filtered at $4 \mathrm{kH}$ using a Multiclamp $700 \mathrm{~B}$ amplified (Molecular Devices) and Im- 
122 Patch@ open access software http://impatch.ifc.unam.mx. Recordings were continued when leak

123 current while $<25$ pA or seal resistance $>1 \mathrm{G} \Omega$. Recordings were analyzed with custom routines

124 in MATLAB.

125 Imaging. To identify the target brain region, we used an upright microscope with a 4X/0.10 NA

126 air objective (Olympus) before switching to 60X/0.90 W NA water immersion objective to confirm

127 ChroME or C1V1 opsin expression on target cells. For ChroME, the mRuby3 fluorescent reporter

128 was excited with monochromatic light transmitted through fiber optics into the microscope

129 (Olympus $100 \mathrm{w}$ high pressure mercury burner model BH2RFLT3). Emitted fluorescence was

130 bandpassed with an Olympus U-49006 ET CY5 filter set: 620/60 ex, 520 dichroic mirror, and

$131700 / 75$ em. For C1V1, the EYFP fluorescent reporter was visualized via a 500/24 excitation filter

132 (Semrock), 520 dichroic mirror (Semrock), and 542/27 emission filter (Semrock). Fluorescence

133 images were acquired (50 ms exposure; $10 \mathrm{~Hz}$ ) using a camera (Orca-ER C4742-95,

134 Hamamatsu) and shutter UNIBLITZ model VCM-D1 controlled by HC Image software

135 (Hamamatsu). We visualized individual neurons' fluorescence deep (100 $\mu \mathrm{m})$ into the brain slice

136 exhibiting a clear L2/3 distinction, taken as evidence of C1V1 or mRuby3 as Cre promoter-driven

137 expression.

138 Photostimulation. Optogenetic photostimulation of ChroMe opsin was performed using a 470

139 nm fiber-coupled LED (M470F1, Thorlabs), fiber optic cannula (M79L01, Thorlabs) and LED driver

140 (M00329012, Thorlabs), while photostimulation of C1V1 was performed using a $617 \mathrm{~nm}$ fiber-

141 coupled LED (M617F2, Thorlabs), fiber optic cannula (CFM14L10, Thorlabs) and LED driver

142 (DC2200, Thorlabs). The photostimulation protocol aimed to mimic stimulation conditions used in

143 previous in vivo experiments (Carrillo-Reid et al., 2016), in order to build artificial photo-

144 ensembles. This photostimulation protocol consisted of trains of $10 \mathrm{~Hz}, 5 \mathrm{~ms}$ light pulses for $4 \mathrm{~s}$

145 followed by a $10 \mathrm{~s}$ rest. 
146 Only neurons that exhibited action potentials or action currents in response to each LED pulse

147 were included in the data analysis. Moreover, LED intensity applied throughout the protocol was

148 the lowest necessary for induce action potentials or action currents. This procedure was carried

149 out for every recording both photostimulated and unstimulated neurons (controls). For

150 photostimulated neurons, the parameters were always monitored before and after ongoing

151 photostimulation protocol (15 to $80 \mathrm{~min}$ ). Photostimulation protocol was stopped every 15 or 20

152 min to monitor the parameters and then it was restarted. For unstimulated neurons, the same

153 parameters were monitored at the beginning and then every $15-20$ min until approximately 80

154 min after no photostimulation.

155 Experimental design. To generate photoensembles (Carrillo-Reid et al., 2016), we delivered 156 Cre-dependent ChroME opsin (Mardinly et al., 2018), with virus injection in transgenic VGlu1-Cre 157 mice, or C1V1 opsin in wild-type mice. No significant differences were found in experiments with 158 both opsins and data were pooled together. Then, we localized the L2/3 pyramidal neurons in 159 primary visual cortex of brain slices expressing ChroME or C1V1 (Figure 1A). Then, in order to 160 test whether the maintenance of an imprinted photo-ensemble was due to reinforcement of 161 synaptic connections among the neurons (Carrillo-Reid et al., 2016) or by cell-intrinsic regulation 162 (Pignatelli et al., 2019), we recorded and characterized passive and active electrical properties of 163 the neurons using whole-cell, cell-attached and perforated patch-clamp recording of at least, two 164 pyramidal neurons simultaneously in vitro (Figure 1B).

165 Once we obtained a patch, we compared cellular electrical properties before and after build the 166 artificial photo-ensembles with the photostimulation protocol. For whole-cell recordings, we 167 examined changes in evoked EPSCs and spontaneous EPSCs (sEPSCs) in response to 168 photostimulation (Figure 1C); for cell-attached recordings we measured changes in spontaneous 169 firing throughout the photostimulation (Figure 1D); for perforated patch clamp we explored 
170 parameters like membrane resistance, rheobase, firing rate and the phase of action potentials

171 (Figure 1E).

172 Statistical Analysis. Statistical details are showing in each figure legends. In general, group data

173 are expressed as mean \pm sem. Shapiro-Wilk was used for normality test. Student's unpaired or

174 paired t test were used for comparing parametric groups. Wilcoxon rank-sum and Mann-Whitney

175 test were used for nonparametric analysis. Differences between two groups were considered

176 significant when ${ }^{*} p<0.05,{ }^{* *} p<0.01$ and ${ }^{* * *} p<0.001$. All statistics were performed using 177 statistical functions in MATLAB. 


\section{Results}

180 Lack of synaptic plasticity between photostimulated neurons

181 Our initial hypothesis, based on our previous results photo-imprinting ensembles in vivo (Carrillo-

182 Reid et al., 2016), was that neuronal ensembles are built by Hebbian synaptic plasticity due to

183 the simultaneous activation of pre and postsynaptic neurons. Thus, new ensembles could be

184 formed by the creation of new synaptic connections or reinforcement or potentiation of existing

185 ones. To explore this we first tested whether persistent and repeated optogenetic activation of

186 connected neurons induced synaptic plasticity in one or both cells (Hebb, 1949; Cossell et al.,

187 2015). In order to answer this question, we performed dual whole-cell recordings from adjacent

188 neurons, which are more likely to be connected (Ko et al., 2011), of L2/3 pyramidal neurons

189 expressing the opsins ChroME or C1V1 in cortical slices from adult animals (Figure 2A). Using

190 patch clamp, we recorded simultaneously from pairs of neurons searching for monosynaptic

191 connections in both directions ( $n=5,4$ mice for photostimulated group and $n=5,3$ mice for

192 unstimulated). For measuring membrane potential, we performed current-clamp recordings,

193 injecting necessary current (between -10 to $10 \mathrm{pA}$ ) to keep neurons at $-70 \mathrm{mV}$, close to their

194 resting membrane potential. Under these conditions, we induced action potentials in presynaptic

195 neurons with 10 current depolarizing pulses at the lowest intensity (amplitude: 400 to 600 pA, 2

$196 \mathrm{~ms}, 20 \mathrm{~Hz}$ trains). For measuring synaptic currents, postsynaptic neurons were recorded in

197 voltage-clamp at a holding potential of $-70 \mathrm{mV}$. This membrane potential prevents the generation

198 of voltage-dependent currents, and better isolate EPSCs. We classified connections as

199 monosynaptic if EPSC latency was < 2 ms relative to its corresponding presynaptic spike (Figure

$2002 B ; n=10,7$ mice). We included in the analysis only cells that exhibited detectable EPSCs at the

201 beginning and end of the recording, which lasted roughly $60 \mathrm{~min}$, including the photostimulation.

202 To confirm the stability in the connection until the end of the recording, we averaged 30 
203 postsynaptic traces, showing 10 EPSCs elicited by the presynaptic train before and after 204 photostimulation for a representative neuron (Figure 2C).

205 In these pairs of connected neurons, we examined if optogenetic photostimulation induced 206 synaptic changes. Visual inspection failed to reveal any major alterations in EPSCs. To quantify 207 this, we calculated the paired-pulse ratio (PPR), i.e. the ratio of amplitudes for the second to the 208 first EPSC in the train $\left(\mathrm{EPSC}_{2} / \mathrm{EPSC}_{1}\right)$ of cells before $(0.7 \pm 0.07 \mathrm{PPR})$ and after photostimulation 209 (0.8 \pm 0.08 PPR; $p=0.2$ Wilcoxon rank-sum; $n=5,4$ mice) and compared it with a control group 210 of unstimulated neurons before $(0.75 \pm 0.03 \mathrm{PPR})$ and after $30 \mathrm{~min}(0.7 \pm 0.1 \mathrm{PPR} ; \mathrm{p}=0.7$

211 Wilcoxon rank-sum; $n=5,3$ mice) (Figure 2D). No significant differences were observed in PPR

212 of evoked EPSC when we compared the changes in PPR of photostimulated $(-0.12 \pm 0.04$, 213 change in PPR) versus unstimulated neurons ( $0.1 \pm 0.1$ change in PPR; $p=0.5$ Mann Whitney's).

214 Moreover, average peak currents of the first EPSC1 (EPSC 1 ) of photostimulated neurons showed 215 no difference before $(24 \pm 9 \mathrm{pA})$ and after photostimulation $(21 \pm 10 \mathrm{pA} ; \mathrm{p}=0.6$ Wilcoxon rank216 sum; $n=5,4$ mice), similarly to unstimulated neurons before $(15 \pm 2 p A)$ and after $30 \min (9 \pm 2$ $217 \mathrm{pA} ; \mathrm{p}=0.06$ Wilcoxon rank-sum; $\mathrm{n}=5,3$ mice). The average change comparison between 218 photostimulated ( $-4 \pm 3 \mathrm{pA}$ change in amplitude) and unstimulated neurons $(-2 \pm 2 \mathrm{pA}$ change in 219 amplitude) were also not statistically different ( $p=0.5$, Mann Whitney's; Figure 2E). We concluded 220 that synaptic plasticity was not altered after photostimulation. Our results suggest that, under our 221 conditions, synaptic plasticity among connected neurons did not contribute to build photo222 ensembles.

\section{Lack of changes in spontaneous synaptic inputs in photostimulated neurons}

225 The previous results did not rule out the possibility that synaptic plasticity among non-connected 226 or non-recorded neurons contributed to the generation of photoensembles. To search for 
227 evidence of synaptic plasticity in the neuronal population, we measured spontaneous EPSCs from 228 recorded neurons and compared them before and after optogenetic stimulation (Figure 3A). Since 229 the viral vector labels pyramidal cells extensively, we reasoned that a significant portion of a given 230 cell's presynaptic partners would also express ChroME or C1V1 and, thus, fire in response to 231 photostimulation. If this leads to synaptic potentiation in the connections, we would expect to 232 detect it as changes in amplitude of spontaneous EPSCs.

233 To test this, we recorded spontaneous EPSCs from connected and unconnected neurons. The 234 changes in mean spontaneous EPSC frequency and amplitude were compared between 235 photostimulated ( $n=12$ pairs, 7 mice) and unstimulated cells ( $n=8$ pairs, 4 mice). We found no 236 statistically difference in spontaneous EPSCs frequency changes between them (-26 $\pm 25 \%$ for 237 photostimulated cells and $-33 \pm 18 \%$ for unstimulated cells; $p=0.4$, Mann Whitney's); or any 238 significant differences in spontaneous EPSCs amplitude ( $-13 \pm 8 \%$ for photostimulated cells and $239-17 \pm 8 \%$ for unstimulated cells; $p=0.9$, Mann Whitney’s) (Figure 3B).

240 We were surprised by that lack of synaptic plasticity after potentiation, and reasoned that synaptic 241 potentiation in connected or unconnected neurons was perhaps masked owing to intracellular 242 washout by prolonged whole-cell recordings. To explore this, we recorded EPSPs using 243 perforated patch clamp from neurons in current clamp, before and after photostimulation, but we 244 also did not observe evidence of new evoked EPSP $(n=6,3$ mice; Figure 3C). Using perforated 245 patch recordings, we also compared the number of spontaneous EPSPs before and after 246 photostimulation (17 \pm 2 EPSPs before and $17 \pm 2$ EPSPs after); instantaneous EPSPs frequency $247(4.5 \pm 0.7 \mathrm{~Hz}$ before and $5.7 \pm 0.6 \mathrm{~Hz}$ after $)$ and EPSPs amplitudes $(1.2 \pm 0.2 \mathrm{mV}$ before and 1.5 $248 \pm 0.2 \mathrm{mV}$ after $)$. None of these parameters showed significant differences $(p=0.4 ; p=0.4$ and $p$ $249=0.7$ respectively; Wilcoxon's test) (Figure 3D). Thus, both whole-cell and perforated recordings 250 showed a similar lack of detectable changes in evoked or spontaneous synaptic inputs after 251 photostimulation. We concluded that, under our experimental conditions, photostimulation of 
252 neurons with the same optogenetic protocols that induce the formation of ensembles in vivo did 253 not generate appreciable changes in synaptic properties.

\section{Optogenetic stimulation increases neuronal spontaneous activity}

256 During our whole cell recordings, we noticed that photostimulated neurons were often 257 spontaneously coactive. Indeed, close inspection of the in vivo data indicated that, after 258 photostimulation, neurons increase their spontaneous activity (Carrillo-Reid et al., 2016). To 259 explore this possibility, we performed cell-attached recordings from neurons in slices to 260 noninvasively investigate the effect of photostimulation on the spontaneous firing rate of neurons.

261 In these experiments, we observed progressive increases in spontaneous firing rate between 262 optogenetic stimuli in $60 \%$ of the neurons $(n=17,5$ mice; Figure $4 A)$. This increase in 263 spontaneous activity persisted for 30 min after photostimulation ( $3 \pm 3$ peaks before vs. $49 \pm 14$ 264 peaks after in $60 \mathrm{~s}$ window; $\mathrm{p}=0.009$; Wilcoxon's test). This suggested that photostimulation 265 increase neuronal excitability and it was not simply due to a rebound response after stimulation 266 (Figure 4B).

267 To analyze with more detail this increase in spontaneous activity, the instantaneous firing 268 frequency was measured in $60 \mathrm{~s}$ windows. We found significant increases in instantaneous 269 frequency after photostimulation $(0.15 \pm 0.13 \mathrm{~Hz}$ before vs. $2 \pm 0.7 \mathrm{~Hz}$ after; $p=0.009$ Wilcoxon's 270 test; $n=17,5$ mice) (Figure 4C, 4D). But for unstimulated neurons, we found no significant 271 differences in number of spikes (17 \pm 8 spikes before vs. $20 \pm 10$ spikes after; $p=0.2$; Wilcoxon's 272 test; $\mathrm{n}=14,2$ mice) or instantaneous frequency $(4 \pm 1.7 \mathrm{~Hz}$ before vs. $5 \pm 1.8 \mathrm{~Hz}$ after; $\mathrm{p}=0.3$

273 Wilcoxon's test; $n=14,2$ mice) at the beginning and end of recording. This increase in 274 spontaneous activity after photostimulation suggests that repeated photostimulation could 275 increase neuronal excitability. 
277 Our results indicated that the formation and stability of a cell ensemble could be mediated by cell278 intrinsic increases in cellular excitability (Ryan et al., 2015; Titley et al., 2017; Abraham et al., 279 2019) rather than by reinforcement of its synapses (Disterhoft and Oh, 2006; Pignatelli et al., 280 2019). To explore cell-intrinsic mechanisms of ensemble formation, we recorded neurons with 281 perforated patch clamp, before and after photostimulation, quantifying intrinsic 282 electrophysiological parameters such as: current injection firing-dependence, membrane 283 resistance, firing rate, frequency and I-V phase plots.

284 We first measured the membrane potential response to current injections with I-V plots. To do 285 this, we kept the membrane potential at $-70 \mathrm{mV}$ and applied series of $500 \mathrm{~ms}$ current injections 286 ranging from $-100 \mathrm{pA}$ to $160 \mathrm{pA}$, at $20 \mathrm{pA}$ increments (Fig. 5A). In a representative neuron, we 287 observed a clear and stable increase in evoked activity after 20 min of photostimulation for lower 288 current steps $(40,60$ and $80 \mathrm{pA})$, with no action potential in the first min with $40 \mathrm{pA}$, and 3 action 289 potentials, 20 min later with the same current injection. Once the increase in response occurred, 290 it did not change, in either direction, for duration of the recording ( 3 action potentials after 80 min 291 of photostimulation with 40 pA of current injection) (Figure 5B).

292 A rise in the excitability could be related to an increase in membrane resistance (Marder and 293 Goaillard, 2006). We used the I-V data with negative current steps to obtain resistance values, 294 before and after photostimulation (Fig. 5C). We observed significant increases in input resistance 295 when comparing the start of the photostimulation ( $293 \pm 32 \mathrm{~m} \Omega ; n=5,4$ mice $)$ of and 30 min later 296 (361 $\pm 30 \mathrm{~m} \Omega ; \mathrm{p}=0.0001 ;$ student's $\mathrm{t}$ test). Meanwhile, resistances measurements in 297 unstimulated neurons between the first minute of IV recording (287 $\pm 65 \mathrm{~m} \Omega)$ and at 30 minutes $298(286 \pm 55 \mathrm{~m} \Omega)$ neurons did not show any changes ( $p=0.9$ student's t test; $n=3,3$ mice; Fig. 5D). 299 We conclude that increases in excitability are associated with increases in membrane resistance. 
301 To measure the physiological effect of the detected changes in membrane excitability in the 302 population of photostimulated neurons, we measured firing rate, mean instantaneous frequency 303 and mean frequency between the two first initial spikes for each current step of the I/V protocol, 304 tabulating all parameters for photostimulated neurons and unstimulated procedures in response 305 to each current step. (Table 1).

To compare firing rates, we averaged the number of spikes before and after photostimulation for each neuron (Fig. 6 A [left panel]). We found that increases in firing rate always occurred after 15 - 20 min of photostimulation at lower current steps (from 40 to $120 \mathrm{pA} ; \mathrm{n}=7,5$ mice). One of the most noticeable changes was found with $60 \mathrm{pA}$ current steps (before: $2.6 \pm 1$ vs. after: $5 \pm 1$

310 spikes; $p=0.009$; student's t test; $n=7,5$ mice). However, no differences were found for highest 311 current steps (140 and $160 \mathrm{pA} ; \mathrm{p}=0.1$ and 0.2 ; student's $\mathrm{t}$ test; $\mathrm{n}=7,5$ mice). Unstimulated 312 neurons did not show differences in firing rate, when we compared the firing rate at minute 1 vs.

31330 minutes later for each current step (from 40 to $160 p A: p>0.05$; student's $t$ test; $n=3,3$ mice)

314 (Fig. 6 A [central panel]). We also found difference in initial firing rate of photostimulated vs.

315 unstimulated neurons (Table 2: for 40 to 80 pA: $p<0.05$; unpaired student's t test; $n=7,5$ mice

316 for photostimulated neurons and $n=3,3$ mice for unstimulated) (Fig. 6 A [right panel]).

317 Similar results were found when analyzing the mean instantaneous firing frequencies, which also 318 increased after photostimulation. The most significant increases happened with 40 to $80 \mathrm{pA}$ 319 current injection for the before and after comparison (see Table 1; $p<0.05$; paired student's $t$ 320 test) (Fig. $6 \mathrm{~B}$ and $\mathrm{C}$ [left panel]). One of the most noticeable changes was with the $60 \mathrm{pA}$ current 321 step (before: $6 \pm 2$ versus after: $10 \pm 2.6 \mathrm{~Hz} ; \mathrm{p}=0.007$; student's $\mathrm{t}$ test; $\mathrm{n}=7,5$ mice). Meanwhile, 322 unstimulated neurons did not show significant differences for lower current steps (40, 60 and 100 $323 \mathrm{pA} ; \mathrm{p}>0.1$; student's t test; $\mathrm{n}=3,3$ mice). However, for higher current steps $(80,120$ to $160 \mathrm{pA})$ 
324 the mean firing frequency significantly decreased, when compared with the data at the first

325 minute. (e.g. $16 \pm 3$ at min 1 vs. $15 \pm 3 \mathrm{~Hz} 30$ min after, for 120 pA step; $p<0.05$; student's t test)

326 (Fig. 6 B [central panel]). We also found significant differences of mean frequency between

327 photostimulated and unstimulated neurons for the lowest current steps (40 and $60 \mathrm{pA}$; see Table

$3282, p<0.01 ;$ unpaired student's t test; $n=7,5$ mice for photostimulated neurons and $n=3,3$ mice

329 for unstimulated) but not for higher current steps (80 to $160 \mathrm{pA} ; \mathrm{p}>0.05$; unpaired student's $\mathrm{t}$ 330 test) (Fig. 6 B [right panel]).

331 Finally, to evaluate the modification of firing properties that could facilitate burst induction, we 332 measured the mean frequency of the two first spikes for each current step. Frequency increases 333 occurred with 40 to 80 pA current steps after photostimulation (see Table 1; $p<0.05$; paired 334 student's t test; $\mathrm{n}=7,5$ mice) (Fig. $6 \mathrm{C}$ [left panel]). The clearest change was for $40 \mathrm{pA}$ current 335 steps, where the mean frequency difference between before and after was $5.3 \pm 1.5 \mathrm{~Hz}(\mathrm{p}=$ $336 \quad 0.001$; paired student's t test). Unstimulated neurons did not show significant differences after 30 337 minutes of recordings for lower current steps (40 to $140 p A ; p>0.05$; paired student's t test). Only 338160 pA current steps resulted in a significant mean frequency decrease (e.g. $42.5 \pm 14$ at min 1 339 vs. $38.5 \pm 14 \mathrm{~Hz} 30 \mathrm{~min}$ after; $p=0.01$; paired student's $\mathrm{t}$ test) (Fig. $6 \mathrm{C}$ [central panel]). We found 340 significant changes in mean frequency between photostimulated and unstimulated neurons for 341 lower current steps (40 and 60 pA; see Table 2; $p<0.01$; unpaired student's t test; $n=7,5$ mice 342 for photostimulated neurons and $n=3,3$ mice for unstimulated), but not for higher current steps 343 (80 to $160 \mathrm{pA} ; \mathrm{p}>0.05$; unpaired student's $\mathrm{t}$ test) (Fig. $6 \mathrm{C}$ [right panel]). This set of results 344 revealed that photostimulation produced a robust increase in current-dependent firing. 


\section{Lowering of firing threshold after photostimulation}

347 Burst firing depends on the interplay between the afterhyperpolarizations (AHPs) and 348 afterdepolarizations (ADPs) that follows the first action potential (Brumberg et al., 2000). To 349 explore this, we evaluated the dynamic of action potentials using phase plot analysis. We used 350 the first spikes from 60 pA current injections in the I-V dataset, because during this current step 351 all neurons displayed action potentials before and after the photostimulation protocol. First, action 352 potentials were detected after setting a threshold of $>0.00015$ in the first derivative of membrane 353 potential waveform, a value which also corresponds to firing threshold. Voltage vs. time values $354(\mathrm{mV} / \mathrm{ms})$ were plotted against the membrane potential $(\mathrm{mV})$. In this analysis, action potentials are represented as a loop in which the starting point represents firing threshold (Fig. 7A). The majority of phase plots display a leftward offset, making evident a shift in firing threshold after 30 min of

357 photostimulation ( $-37 \pm 3$ before vs. $-42.7 \pm 3 \mathrm{mV}$ after; $\mathrm{p}=0.008$; Wilcoxon' test; $\mathrm{n}=8,5$ mice).

358 Consistent with this, we found a significant reduction in firing threshold before and after 359 photostimulation ( $3.5 \pm 1 \mathrm{mV} ; \mathrm{p}=0.0078$ by Wilcoxon's test; $\mathrm{n}=8$ cells, 5 mice $)$. Analogous to 360 long lasting changes in firing rate, the reduction in firing threshold was stable and did not increases 361 more even $40 \mathrm{~min}$ after photostimulation protocol $(3.7 \pm 1 \mathrm{mV}$ after $30 \mathrm{~min}$ vs. $4.2 \pm 1$ after 40 $362 \min ; p=0.9 ;$ Wilcoxon's test; $n=8,5$ mice). Meanwhile, we found no statistically significant 363 differences in firing threshold of unstimulated neurons, when comparing the beginning versus the 364 end of recording ( $-40 \pm 3$ and $-39 \pm 3 \mathrm{mV}$ respectively; $p=0.25 ; n=3$ cells, 3 mice) (Figure 7B).

365 We also documented a lowering of the threshold voltage when we compared the change in 366 threshold voltage at the first minute $(0.4 \pm 0.2 \mathrm{mV}), 30 \mathrm{~min}(3.7 \pm 1 \mathrm{mV} ; \mathrm{p}=0.0078)$ and $40 \mathrm{~min}$ 367 (4.2 $\pm 1 \mathrm{mV} ; \mathrm{p}=0.03$ by Wilcoxon's test; $\mathrm{n}=8$ cells) for photostimulated cells. Unstimulated 368 neurons displayed no changes $(0.6 \pm 0.4 \mathrm{mV}$ at the first min vs. $-0.8 \pm 0.5 \mathrm{mV} 30 \mathrm{~min}$ later; $p=$ 369 0.25; Wilcoxon's test; $n=3,3$ mice) (Figure 7C). These decreases in threshold for 
370 photostimulated neurons were significant, when compared to unstimulated neurons $(3.7 \pm 1 \mathrm{mV}$

371 photostim vs. $-0.8 \pm 0.5 \mathrm{mV}$ unstimulated neurons; $p=0.25$; Mann Whitney's test).

372 Altogether, these results indicate that photostimulation significantly increases membrane

373 resistance and lowers firing threshold, which increases action potential firing. These effects nicely

374 explain the increase in neuronal excitability previously observed (Figure 4). Thus,

375 photostimulation could enable weak synaptic inputs to reach firing threshold, facilitating the

376 formation or maintenance of a neuronal ensemble. 


\section{Discussion}

379 The traditional view of a neuronal ensemble is that it is created by strengthening of its synaptic 380 connections after repeated coactivation (Hebb, 1949; Carrillo-Reid et al., 2016; Hoshiba et al., 381 2017). However, recent results have questioned whether synaptic plasticity is the unique 382 mechanism of neuronal ensembles formation (Zhang and Linden, 2003; Disterhoft and Oh, 2006; 383 Ryan et al., 2015). Consistent with this, here we describe that the co-photostimulation of pairs of 384 connected or unconnected neurons, the same manipulation that builds an artificial neuronal 385 ensemble in vivo (Carrillo-Reid et al., 2016), do not induce detectable changes in synaptic 386 plasticity. Instead, neurons become more active spontaneously after photostimulation, apparently 387 due to increases in membrane resistance and reductions in action potential threshold, as cell388 intrinsic mechanisms. Thus, neuronal excitability may increase the efficiency of existing synaptic 389 connections between neurons in an ensemble, without necessarily altering synaptic plasticity.

391 Role of synaptic mechanisms in ensemble formation

392 The formation or maintenance of neuronal ensembles have been ascribed to increases in synaptic 393 connectivity (Hebb, 1949; Carrillo-Reid et al., 2016; Hoshiba et al., 2017). Indeed, there is a 394 preferential connectivity between neurons that corresponds with similar receptive fields (Cossell 395 et al., 2015). An increased connectivity also probably underlies pattern completion in cortical 396 ensembles (Carrillo-Reid et al., 2019). Thus, a few strong connections could drive local excitation 397 of the majority of neurons with weak connections (Cossell et al., 2015). Here we mimic in vitro 398 previous two-photon optogenetic photostimulation experiments in vivo to generate ensembles in 399 layer 2/3 pyramidal cells from mouse primary visual cortex (Carrillo-Reid et al., 2016), and tested 400 whether synaptic mechanisms were involved in ensemble formation. We measured the amplitude 
401 of evoked EPSC and PPR to examine whether synaptic efficiency between two connected

402 neurons had changed (Bliss and Gardner-Medwin, 1973).

403 Disproving our initial hypothesis, our findings surprisingly indicated a lack of significant changes

404 in synaptic parameters of connected neurons and we did not detect any new connections formed

405 after photostimulation in previously unconnected neurons. Moreover, spontaneous synaptic

406 inputs apparently also remained the same. Although we did not observe significant differences in

407 any case, in spite of changes in synaptic plasticity being our initial hypothesis based on our 408 previous in vivo data (Carrillo-Reid et al., 2016), it is possible that the in vitro conditions could

409 have obscured these changes. A lack of synaptic plasticity may also be due to a suppression of

410 neurotransmitter release (Fioravante and Regehr, 2011). Certainly, prolonged presynaptic

411 stimulation could induce short-term synaptic depression (Schneggenburger et al., 2002). In any

412 case, the heterogeneity have found in synaptic facilitation and depression, as seen in our data,

413 matches other published reports (Schneggenburger et al., 2002). But because of these negative

414 results, we suspected that mechanisms other than synaptic plasticity could be participating in the

415 artificial ensemble consolidation or formation.

\section{Cell-intrinsic mechanisms of ensemble formation}

418 Indeed, although no changes were observed in synaptic parameters, robust activity-dependent

419 increases in spontaneous firing were easily detected in photostimulated neurons. This was 420 consistent with increases in spontaneous activity observed with calcium signals after 421 photostimulation training in vivo (Carrillo-Reid et al., 2016). This suggests that the in vivo 422 photostimulation protocol was accurately reproduced in our in vitro conditions. 
423 An increase in cellular activity through cell-intrinsic mechanisms after prolonged firing is

424 consistent with many functional studies in vertebrates, even under very different experimental

425 protocols. For example: increases in excitability are found following LTP induction in visual cortex

426 (Cudmore and Turrigiano, 2004) or in hippocampal CA1 (Xu, 2005), and after behavior training in

427 CA1 (Disterhoft et al., 1986). Intrinsic excitability is also modified when an animal is exposed to

428 novel sensorial experience (Brown et al., 2019) or after environmental enrichment (Valero-

429 Aracama et al., 2015). Our results reveal an additional paradigm to induce a similar increase in

430 spontaneous activity by direct postsynaptic photostimulation and imply that optogenetic imprinting

431 of ensembles (Carrillo-Reid et al., 2016), increases postsynaptic excitability.

\section{Modification of Intrinsic mechanisms by photostimulation}

434 Our data reveal an intensity-dependent and persistent increase in firing rate after 435 photostimulation. This may be due to the increase in membrane resistance and/or the decreases

436 in rheobase that we observed. The synergy between these two mechanisms could make the 437 neuron reach its firing threshold with smaller depolarizations. Thus, an increase in excitability is 438 particularly relevant for lower current injections generated by weaker inputs. We propose that, 439 after photostimulation, neurons shift to a more excitable state, revealing new connections, like an 440 iceberg emerging out of the water (Figure 7). Therefore the same, unchanged synaptic inputs 441 could bring neurons to threshold and induce increased output firing after photostimulation, 442 improving their synaptic efficiency in postsynaptic pyramidal neurons (Zhang and Linden, 2003; 443 Titley et al., 2017; Lisman et al., 2018; Debanne et al., 2019). Neuronal photostimulation would 444 functionally alter the circuit, but without changing the synapses themselves and this phenomenon 445 could occur very rapidly. 
446 Modifications in sub- and suprathreshold membrane conductance that initiate an action potential

447 or a burst could underlie increased excitability (Brumberg et al., 2000). In terms of passive

448 properties, our results coincide with many others studies that have shown alterations in input

449 resistance as the mechanism that can be responsible for increases in excitability (Disterhoft et

450 al., 1986; Valero-Aracama et al., 2015; Brown et al., 2019; Pignatelli et al., 2019). Activity-

451 dependent increase in input resistance have been shown in hippocampal neurons, where it has

452 been proposed that the larger increase in excitability could be potentiated by a suppression of $G$

453 protein-couple inwardly rectifying $\mathrm{K}^{+}$channels (GIRK channels) (Valero-Aracama et al., 2015); or

454 by cAMP-responsive element-binding protein (CREB)-dependent control of excitability by

455 reducing $\mathrm{K}^{+}$conductance (Yu et al., 2017; Lisman et al., 2018). Moreover, recent studies show

456 that persisting firing could be mediated by an ether-à-gogo related gene (ERG) $\mathrm{K}^{+}$channel in

457 neocortical pyramidal neurons (Cui and Strowbridge, 2018; Debanne et al., 2019). In this view,

458 calcium entry induced by repetitive activation could modulate leak potassium currents in the

459 neurons, which will ease their persistent activity.

460 Reported cell-intrinsic mechanisms that induce excitability seem to be cellular-type and perhaps 461 also stimulation protocol-dependent (Angelo et al., 2012). Hence, diverse changes in action 462 potential firing have been identified. One of the most common changes is a reduction in after463 hyperpolarization (AHP) amplitude, described in hippocampal neurons (Disterhoft et al., 1986; 464 Zhang and Linden, 2003; Disterhoft and Oh, 2006; Malik and Chattarji, 2012; Pignatelli et al., 465 2019). Nevertheless, we found a significant decrease in spike threshold as the most robust 466 change in photostimulated neurons. This parameter also determines the firing frequency of 467 neurons associated with voltage-dependent sodium or calcium currents. Changes in threshold 468 appears to be common in cortical neurons after intrinsic plasticity induction (Cudmore and 469 Turrigiano, 2004; Paz et al., 2009), but has also been found in hippocampal neurons (Xu, 2005; 470 Malik and Chattarji, 2012; Valero-Aracama et al., 2015). Consistent with this, visual deprivation 
471 increases spike threshold in pyramidal visual cortex, as an example of a mechanism that reduces

472 neuronal excitability (Brown et al., 2019). It has been proposed that a regulation of the persistent

473 sodium current could underlie the lowering of spike threshold, modulated by protein kinase C

474 (Astman et al., 1998; Valero-Aracama et al., 2015), or protein kinase A activation-dependent of

475 calcium influx (Cudmore and Turrigiano, 2004). Therefore, the intrinsic plasticity by optogenetic

476 photostimulation that we report in L2/3 pyramidal neurons from visual cortex could result from

477 modifications of voltage-gated ion channels and inward rectification by potassium channels.

478 Future studies will examine the exact molecular mechanisms underlying these increases in 479 excitability.

481 Intrinsic excitability in neuronal ensembles formation

482 Many studies have shown that Hebbian plasticity is required for intrinsic plasticity induction 483 (Debanne et al., 2019). Thus, NMDA receptor activation is necessary to increase firing probability 484 in neurons (Disterhoft et al., 1986; Cudmore and Turrigiano, 2004; Xu, 2005; Malik and Chattarji, 485 2012; Kim et al., 2016; Pignatelli et al., 2019). Behavioral training also induce neuronal excitability 486 through Hebbian mechanisms (Disterhoft et al., 1986; Malik and Chattarji, 2012; Valero-Aracama 487 et al., 2015). Other studies, agreeing with our results, have demonstrated that for increasing 488 neuronal excitability, postsynaptic stimulation could be enough to improve synaptic efficiency 489 (Stackman et al., 2002; Paz et al., 2009).

490 Our work provides evidence that supports the relevance of intrinsic mechanisms induced by 491 photostimulation training in neuronal ensemble formation. The possibility to change the output 492 pattern of a postsynaptic neuron through the modification of its firing properties could be a factor 493 that promotes synaptic efficacy (Lisman, 1997; Brumberg et al., 2000). Thus, fast plasticity in 


\section{Alejandre-García et al., p.24}

494 intrinsic excitability could have a major impact on network dynamics and could serve as an

495 important information-storage mechanism which may contribute to memory formation (Xu, 2005;

496 Marder and Goaillard, 2006; Lisman et al., 2018; Pignatelli et al., 2019) and to the generation of

497 intrinsic circuit states, such as ensembles and attractors (Hopfield, 1982; Buzsáki, 2010; Miller et

498 al., 2014b; Carrillo-Reid et al., 2016, 2019). 


\section{References}

Abeles M (1991) Corticonics. Cambridge University Press.

Abraham WC, Jones OD, Glanzman DL (2019) Is plasticity of synapses the mechanism of longterm memory storage? npj Sci Learn 4:9.

Angelo K, Rancz EA, Pimentel D, Hundahl C, Hannibal J, Fleischmann A, Pichler B, Margrie TW (2012) A biophysical signature of network affiliation and sensory processing in mitral cells. Nature 488:375-378.

Astman N, Gutnick MJ, Fleidervish IA (1998) Activation of Protein Kinase C Increases Neuronal Excitability by Regulating Persistent $\mathrm{Na}+$ Current in Mouse Neocortical Slices. J Neurophysiol 80:1547-1551.

Bliss TVP, Gardner-Medwin AR (1973) Long-lasting potentiation of synaptic transmission in the dentate area of the unanaesthetized rabbit following stimulation of the perforant path. $J$ Physiol 232:357-374.

Brown APY, Cossell L, Margrie TW (2019) Visual Experience Regulates the Intrinsic Excitability of Visual Cortical Neurons to Maintain Sensory Function. Cell Rep 27:685-689.e4.

Brumberg JC, Nowak LG, McCormick DA (2000) lonic Mechanisms Underlying Repetitive HighFrequency Burst Firing in Supragranular Cortical Neurons. J Neurosci 20:4829-4843.

Buzsáki G (2010) Neural syntax: cell assemblies, synapsembles, and readers. Neuron 68:362385.

Carrillo-Reid L, Han S, Yang W, Akrouh A, Yuste R (2019) Controlling Visually Guided Behavior

Carrillo-Reid L, Yang W, Bando Y, Peterka DS, Yuste R (2016) Imprinting and recalling cortical

523 Churchland PS, Sejnowski TJ (1992) The computational brain. Cambridge, MA, US: The MIT Press.

525 Cossart R, Aronov D, Yuste R (2003) Attractor dynamics of network UP states in the neocortex. Nature 423:283-288.

527 Cossell L, lacaruso MF, Muir DR, Houlton R, Sader EN, Ko H, Hofer SB, Mrsic-Flogel TD (2015) 528 Functional organization of excitatory synaptic strength in primary visual cortex. Nature 518:399-403. 
Cudmore RH, Turrigiano GG (2004) Long-Term Potentiation of Intrinsic Excitability in LV Visual Cortical Neurons. J Neurophysiol 92:341-348.

532 Cui ED, Strowbridge BW (2018) Modulation of Ether-à-Go-Go Related Gene (ERG) Current 533 Governs Intrinsic Persistent Activity in Rodent Neocortical Pyramidal Cells. J Neurosci 534 38:423-440.

Debanne D, Inglebert Y, Russier M (2019) Plasticity of intrinsic neuronal excitability. Curr Opin Neurobiol 54:73-82.

Disterhoft JF, Coulter DA, Alkon DL (1986) Conditioning-specific membrane changes of rabbit hippocampal neurons measured in vitro. Proc Natl Acad Sci 83:2733-2737.

Disterhoft JF, Oh MM (2006) Learning, aging and intrinsic neuronal plasticity. Trends Neurosci 29:587-599.

Fioravante D, Regehr WG (2011) Short-term forms of presynaptic plasticity. Curr Opin Neurobiol 21:269-274.

Hamm JP, Peterka DS, Gogos JA, Yuste R (2017) Altered Cortical Ensembles in Mouse Models of Schizophrenia. Neuron 94:153-167.e8.

545 Hebb DO (1949) The Organization of Behavior, New York: Wiley.

546 Hopfield JJ (1982) Neural networks and physical systems with emergent collective computational abilities. Proc Natl Acad Sci U S A 79:2554-2558.

548 Hoshiba Y, Wada T, Hayashi-Takagi A (2017) Synaptic Ensemble Underlying the Selection and 549 Consolidation of Neuronal Circuits during Learning. Front Neural Circuits 11.

550 Johansson F, Jirenhed D-A, Rasmussen A, Zucca R, Hesslow G (2014) Memory trace and 551 timing mechanism localized to cerebellar Purkinje cells. Proc Natl Acad Sci 111:1493055214934.

553 Kim T, Oh WC, Choi JH, Kwon H-B (2016) Emergence of functional subnetworks in layer 2/3 $554 \quad$ cortex induced by sequential spikes in vivo. Proc Natl Acad Sci 113:E1372-E1381.

555 Ko H, Hofer SB, Pichler B, Buchanan KA, Sjöström PJ, Mrsic-Flogel TD (2011) Functional 556 specificity of local synaptic connections in neocortical networks. Nature 473:87-91.

557 Lisman J, Cooper K, Sehgal M, Silva AJ (2018) Memory formation depends on both synapse558 specific modifications of synaptic strength and cell-specific increases in excitability. Nat $559 \quad$ Neurosci 21:309-314. 
Lisman JE (1997) Bursts as a unit of neural information: making unreliable synapses reliable. Trends Neurosci 20:38-43.

Lorente de Nó R (1933) Studies on the structure of the cerebral cortex. I. The area entorhinalis. $\mathrm{J}$ für Psychol und Neurol.

Lorente De Nó R (1938) Analysis of the activity of the chains of internuncial neurons. J Neurophysiol 1:207-244.

Malik R, Chattarji S (2012) Enhanced intrinsic excitability and EPSP-spike coupling accompany enriched environment-induced facilitation of LTP in hippocampal CA1 pyramidal neurons. J Neurophysiol 107:1366-1378.

Marder E, Goaillard J-M (2006) Variability, compensation and homeostasis in neuron and network function. Nat Rev Neurosci 7:563-574.

Mardinly AR, Oldenburg IA, Pégard NC, Sridharan S, Lyall EH, Chesnov K, Brohawn SG, Waller L, Adesnik H (2018) Precise multimodal optical control of neural ensemble activity.

Marshel JH, Kim YS, Machado TA, Quirin S, Benson B, Kadmon J, Raja C, Chibukhchyan A, Ramakrishnan C, Inoue M, Shane JC, McKnight DJ, Yoshizawa S, Kato HE, Ganguli S,

Miller J -e. K, Ayzenshtat I, Carrillo-Reid L, Yuste R (2014a) Visual stimuli recruit intrinsically generated cortical ensembles. Proc Natl Acad Sci 111:E4053-E4061.

Miller JK, Ayzenshtat I, Carrillo-Reid L, Yuste R (2014b) Visual stimuli recruit intrinsically generated cortical ensembles. Proc Natl Acad Sci U S A 111:E4053-61.

Paz JT, Mahon S, Tiret P, Genet S, Delord B, Charpier S (2009) Multiple forms of activitydependent intrinsic plasticity in layer V cortical neurones in vivo. J Physiol 587:3189-3205.

Pignatelli M, Ryan TJ, Roy DS, Lovett C, Smith LM, Muralidhar S, Tonegawa S (2019) Engram Cell Excitability State Determines the Efficacy of Memory Retrieval. Neuron 101:274284.e5.

Ryan TJ, Roy DS, Pignatelli M, Arons A, Tonegawa S (2015) Engram cells retain memory under retrograde amnesia. Science (80- ) 348:1007-1013. 
591 Schüz A, Braitenberg V (2001) Cerebral Cortex: Organization and Function. In: International

592 Encyclopedia of the Social \& Behavioral Sciences, pp 1634-1640. Elsevier.

593 Shepherd GM (2004) The Synaptic Organization of the Brain (Shepherd GM, ed). Oxford

$594 \quad$ University Press.

595 Stackman RW, Clark AS, Taube JS (2002) Hippocampal spatial representations require 596 vestibular input. Hippocampus 12:291-303.

597 Ting JT, Lee BR, Chong P, Soler-Llavina G, Cobbs C, Koch C, Zeng H, Lein E (2018)

598 Preparation of Acute Brain Slices Using an Optimized <em>N</em>-Methyl-D-glucamine $599 \quad$ Protective Recovery Method. J Vis Exp:e53825.

600 Titley HK, Brunel N, Hansel C (2017) Toward a Neurocentric View of Learning. Neuron 95:19_ 60132.

602 Tonegawa S, Pignatelli M, Roy DS, Ryan TJ (2015) Memory engram storage and retrieval. Curr 603 Opin Neurobiol 35:101-109.

604 Valero-Aracama MJ, Sauvage MM, Yoshida M (2015) Environmental enrichment modulates 605 intrinsic cellular excitability of hippocampal CA1 pyramidal cells in a housing duration and 606 anatomical location-dependent manner. Behav Brain Res 292:209-218.

607 Xu J (2005) Activity-Dependent Long-Term Potentiation of Intrinsic Excitability in Hippocampal 608 CA1 Pyramidal Neurons. J Neurosci 25:1750-1760.

609 Yu X-W, Oh MM, Disterhoft JF (2017) CREB, cellular excitability, and cognition: Implications for $610 \quad$ aging. Behav Brain Res 322:206-211.

611 Yuste R (2015) From the neuron doctrine to neural networks. Nat Rev Neurosci 16:487-497.

612 Zhang W, Linden DJ (2003) The other side of the engram: experience-driven changes in 613 neuronal intrinsic excitability. Nat Rev Neurosci 4:885-900. 
Table 1. Comparation of parameters associated with membrane excitability in intensity-depended response, for photostimulated and unstimulated neurons.

\begin{tabular}{|c|c|c|c|c|c|c|c|c|c|}
\hline \multirow[b]{2}{*}{$\mathrm{pA}$} & \multicolumn{3}{|c|}{ Number of spikes } & \multicolumn{3}{|c|}{$\begin{array}{l}\text { Mean instant firing frequencies } \\
(\mathrm{Hz})\end{array}$} & \multicolumn{3}{|c|}{$\begin{array}{l}\text { Mean frequencies of two first } \\
\text { spikes }(\mathrm{Hz})\end{array}$} \\
\hline & Before & After & P Value & Before & After & P Value & Before & After & P Value \\
\hline \multicolumn{10}{|c|}{ Photostimulated neurons } \\
\hline 20 & 0.0 & 0.0 & - & 0.0 & 0.0 & - & 0.0 & 0.0 & - \\
\hline 40 & $0 \pm 0.07$ & $2 \pm 1$ & $0.01^{* *}$ & 0 & $7 \pm 1.9$ & $0.001 * * *$ & $0 \pm 0$ & $7.4 \pm 2$ & $0.001 * * *$ \\
\hline 60 & $2.6 \pm 1$ & $5 \pm 1$ & 0.009 ** & $6 \pm 2$ & $10 \pm 2.6$ & 0.007 ** & $7 \pm 3$ & $14 \pm 6$ & $0.006 * *$ \\
\hline 80 & $5 \pm 1$ & $7 \pm 0.8$ & 0.009 ** & $11 \pm 2.8$ & $14.6 \pm 3$ & 0.04 * & $14 \pm 6$ & $21 \pm 8$ & 0.05 * \\
\hline 100 & $7 \pm 2$ & $8 \pm 0.8$ & 0.03 * & $15.4 \pm 3$ & $17.4 \pm 3$ & 0.06 & $25 \pm 11$ & $29.4 \pm 15$ & 0.1 \\
\hline 120 & $8 \pm 1.3$ & $9 \pm 1$ & 0.04 * & $18 \pm 4.6$ & $20 \pm 3.7$ & 0.3 & $37 \pm 20$ & $36.6 \pm 18$ & 0.9 \\
\hline 140 & $9 \pm 1.6$ & $10.4 \pm 1$ & 0.1 & $22 \pm 4$ & $23 \pm 3$ & 0.6 & $51 \pm 22$ & $49 \pm 21$ & 0.6 \\
\hline 160 & $9.8 \pm 1$ & $11 \pm 0.5$ & 0.2 & $24.2 \pm 5$ & $24.8 \pm 4$ & 0.7 & $57 \pm 28$ & $57 \pm 28$ & 0.9 \\
\hline
\end{tabular}

Unstimulated neurons

\begin{tabular}{l|lll|lll|lll}
20 & 0.0 & 0.0 & - & 0.0 & 0.0 & - & 0.0 & 0.0 & - \\
40 & $1 \pm 2$ & $1 \pm 2$ & 1 & $2.4 \pm 4.2$ & $2.2 \pm 3.8$ & 0.4 & $2.7 \pm 5$ & $2.4 \pm 4$ & 0.4 \\
60 & $3 \pm 2$ & $2 \pm 2$ & 0.4 & $5.7 \pm 5.7$ & $4.8 \pm 5$ & 0.2 & $7 \pm 8$ & $6 \pm 7$ & 0.2 \\
80 & $5 \pm 2$ & $4 \pm 2$ & 1 & $10 \pm 4$ & $9.3 \pm 4.4$ & $0.03 *$ & $14 \pm 7.4$ & $12.4 \pm 7$ & $0.02 *$ \\
100 & $6 \pm 2$ & $6 \pm 1.5$ & 0.4 & $13.6 \pm 4$ & $13 \pm 4.7$ & 0.3 & $20 \pm 8.6$ & $21 \pm 11.6$ & 0.8 \\
120 & $7 \pm 1.8$ & $6.3 \pm 2$ & 0.2 & $16 \pm 5$ & $15 \pm 5$ & $0.03 *$ & $28.6 \pm 15$ & $24.7 \pm 13$ & 0.1 \\
140 & $8 \pm 2$ & $7.7 \pm 2$ & 0.4 & $19 \pm 5.5$ & $16.7 \pm 6$ & $0.02 *$ & $35 \pm 20.5$ & $32 \pm 21$ & 0.2 \\
160 & $9 \pm 2$ & $8.3 \pm 2$ & 0.2 & $21 \pm 6$ & $19 \pm 7$ & $0.03 *$ & $43 \pm 25$ & $39 \pm 24.6$ & 0.01 ** \\
\hline
\end{tabular}

617 Table 1. Firing rate at minute 1 vs. 30 minutes later for each current step for the number of spikes,

618 mean instant firing frequencies, mean frequencies of two first spikes. Comparation to determine

619 the change in intensity-depended response in photostimulated ( $n=7,5$ mice) and unstimulated

620 condition $\left(n=3,3\right.$ mice). Data represent mean \pm SEM. ${ }^{*} p<0.05,{ }^{* *} p<0.01$ and ${ }^{* * *} p<0.001$ by

621 student's t test. 
Table 2. Comparation of the changes in intensity-depended response for photostimulated vs. unstimulated neurons.

\begin{tabular}{|c|c|c|c|c|c|c|c|c|c|}
\hline \multirow[b]{2}{*}{$\mathrm{pA}$} & \multicolumn{3}{|c|}{ Number of spikes } & \multicolumn{3}{|c|}{ Mean instant firing frequencies $(\mathrm{Hz})$} & \multicolumn{3}{|c|}{$\begin{array}{l}\text { Mean frequencies of two first spikes } \\
(\mathrm{Hz})\end{array}$} \\
\hline & Photostim & Unstim & P Value & Photostim & Unstim & P Value & Photostim & Unstim & P Value \\
\hline 20 & 0.0 & 0.0 & - & 0.0 & 0.0 & - & 0.0 & 0.0 & - \\
\hline 40 & $1.9 \pm 1$ & $0 \pm 0$ & 0.04 * & $7 \pm 0.4$ & $-0.2 \pm 2$ & $0.0006^{* * *}$ & $-0.3 \pm 0.5$ & $7.4 \pm 2$ & 0.0006 *** \\
\hline 60 & $2.3 \pm 2$ & $-0.3 \pm 0.5$ & 0.02 * & $3.4 \pm 0.8$ & $-1 \pm 2$ & $0.009 * *$ & $-1 \pm 1$ & $4.7 \pm 2.5$ & $0.007^{* *}$ \\
\hline 80 & $2 \pm 1$ & $-1 \pm 0$ & $0.007 * *$ & $3.7 \pm 0.3$ & $-1 \pm 4$ & 0.07 & $-1.5 \pm 0.3$ & $6.8 \pm 7.3$ & 0.09 \\
\hline 100 & $1 \pm 1$ & $-0.3 \pm 0.5$ & 0.06 & $2 \pm 1$ & $-0.8 \pm 2.3$ & 0.08 & $0.7 \pm 5.5$ & $4.3 \pm 6.4$ & 0.4 \\
\hline 120 & $1 \pm 1$ & $-0.6 \pm 0.5$ & $0.02 *$ & $1.5 \pm 0.3$ & $-1 \pm 3$ & 0.1 & $-3.9 \pm 2.6$ & $-0.3 \pm 8.5$ & 0.5 \\
\hline 140 & $1.2 \pm 1$ & $-0.3 \pm 0.5$ & 0.1 & $0.6 \pm 0.6$ & $-2 \pm 2.7$ & 0.1 & $-3 \pm 3.1$ & $-2.5 \pm 9.7$ & 0.9 \\
\hline 160 & $1 \pm 1$ & $-0.6 \pm 0.5$ & 0.1 & $0.5 \pm 0.5$ & $-2 \pm 3.2$ & 0.2 & $-4 \pm 0.7$ & $-0.4 \pm 10$ & 0.5 \\
\hline
\end{tabular}

623 Table 2. Mean differences, at the first minute and 30 minutes after, for the number of spikes,

624 mean instant firing frequencies, mean frequencies of two first spikes. Comparation of the changes

625 in the intensity-depended for photostimulated response ( $n=7,5$ mice) vs. unstimulated condition

$626\left(n=3,3\right.$ mice). Data represent mean \pm SEM. ${ }^{*} p<0.05,{ }^{* *} p<0.01$ and ${ }^{* * *} p<0.001$ by unpaired

627 student's t test. 

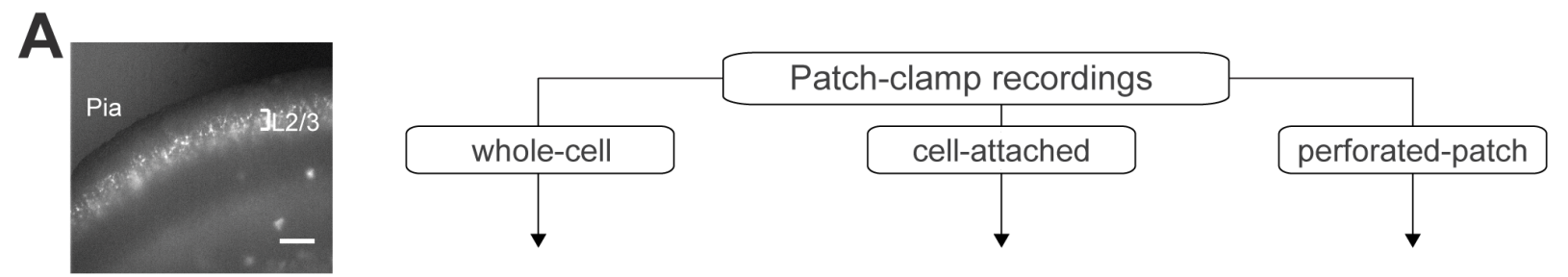

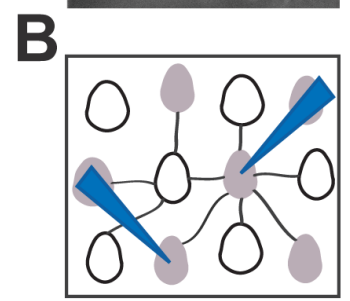

In vitro pair recordings

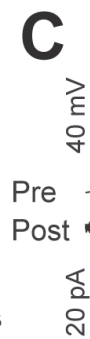

Figure 1. Experimental design.

(A). Example image of Ruby3 reporter fluorescent of ST-ChroMe opsin expression in L2/3 pyramidal neurons in primary visual cortex. Sagittal brain slices were obtained from virus injected

634 mice. Scale bar: $200 \mu \mathrm{M}$. (B) Illustration of In vitro pair recording for evaluating monosynaptic

635 connectivity between neurons. (C) Whole-cell recording of presynaptic action potentials elicited

636 by $20 \mathrm{~Hz}$ train of 10 current injections ( 2 ms each), followed by the identification of a monosynaptic

637 connection, with postsynaptic currents corresponding to presynaptic spikes. (D) Cell-attached and

638 perforated current-clamp recording (E) at the beginning of direct optogenetic photostimulation 639 protocol: 1 to $40 \mathrm{~min}$ of $10 \mathrm{~Hz}$ train, $5 \mathrm{~ms}$ light pulses for $4 \mathrm{~s}$ followed by $10 \mathrm{~s}$ of rest. 
A
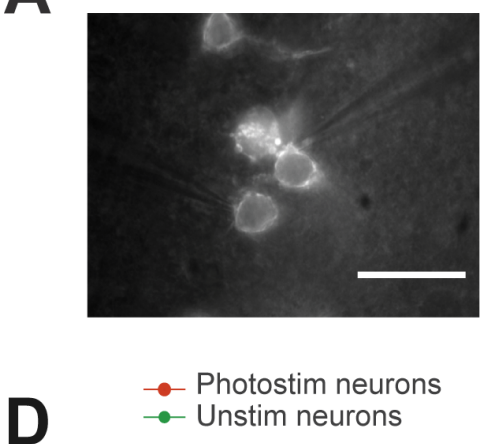
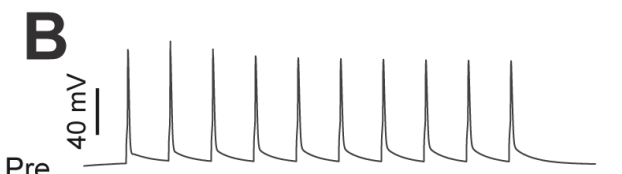

Post

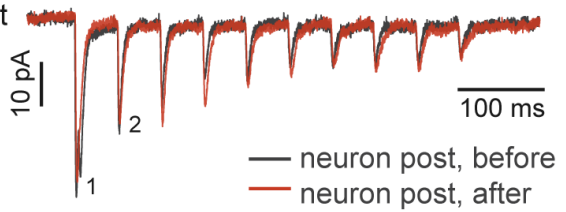

E
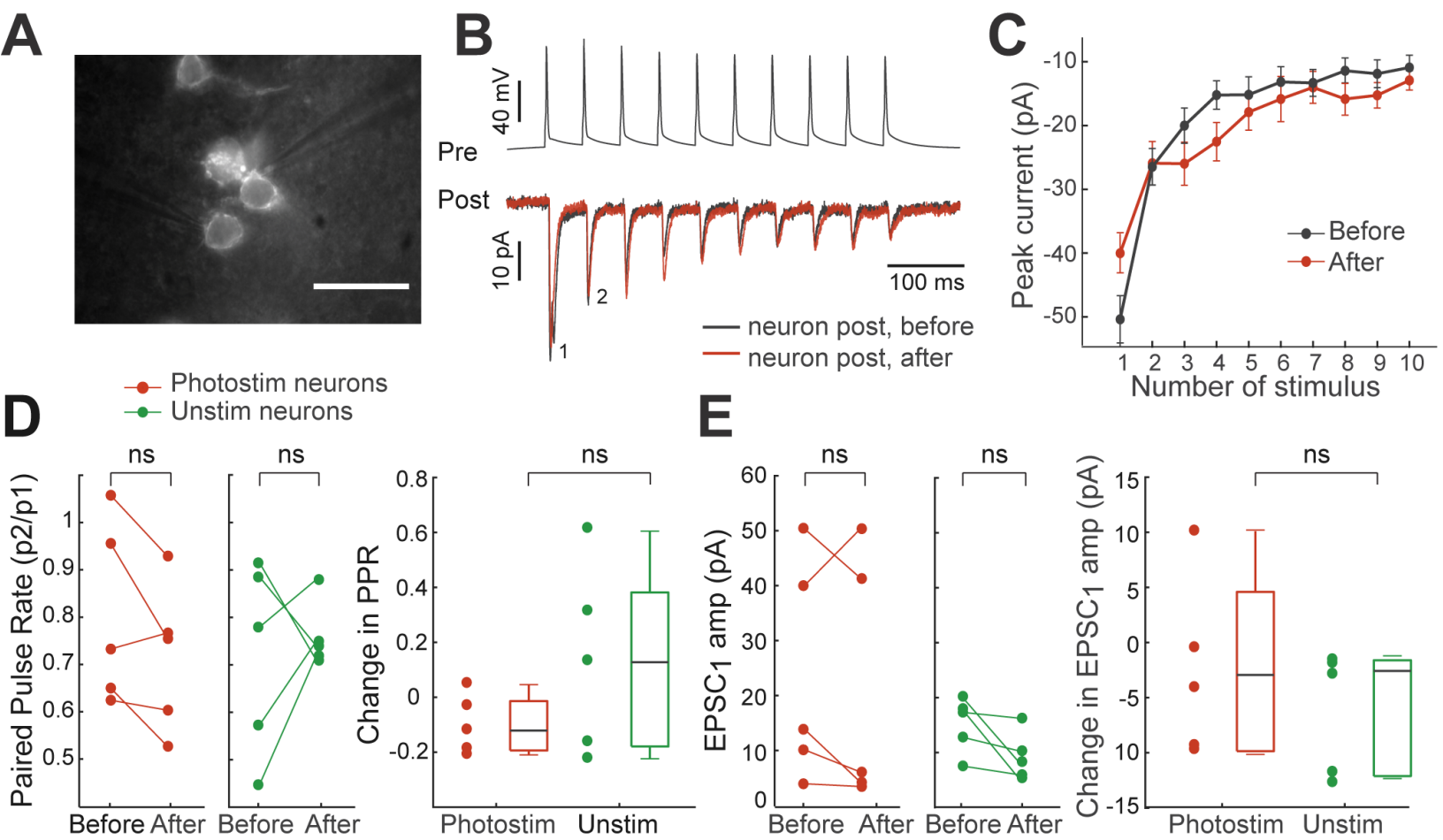

Figure 2. Lack of effect of photostimulation on evoked synaptic inputs

644 (A) Paired whole-cell recording of presynaptic and postsynaptic neurons during photostimulation.

645 Fluorescent reporter expression of ST-ChroMe opsin in pyramidal neurons L2/3 of primary visual cortex from a mouse brain slice (p40). Scale bar: $40 \mu \mathrm{M}$. (B) Representative paired recording of synaptically connected neurons. Top: current-clamp recording of presynaptic action potentials in response to 10 pulses (2 ms each) at $20 \mathrm{~Hz}$ of current injection. Bottom: voltage-clamp recording of evoked EPSCs before (black) and after (red) 40 min of photostimulation. Each trace is the

650 average of 30 successive responses evoked by presynaptic electrical stimulation. (C) Amplitude 651 of evoked EPSCs remain unchanged after optogenetic photostimulation. Average amplitude of 65230 successive EPSCs evoked in the representative neuron in B, before (black) and after (red) 653 photostimulation. $p=0.7$ by Student's $t$ test. Data represent mean \pm SEM. (D) Mean Paired Pulse 654 Ratio (PPR) remains unchanged before and after photostimulation (red; $p=0.2$ by Wilcoxon's test; $n=5$ cells, 4 mice) and unstimulated neurons (green; $p=0.7$ by Wilcoxon's test, $n=5$ cells, 
6563 mice). Right: comparison of change in mean PPRs plotted raw and as a box and whisker plot.

$657 \mathrm{p}=0.5$ by Mann Whitney's test; $\mathrm{n}=5$ cells per group. (E) Mean EPSC $_{1}$ amplitude without

658 significant changes before and after photostimulation protocol (red; $p=0.6$ by Wilcoxon's test; $n$

$659=5$ cells) and unstimulated neurons (green; $\mathrm{p}=0.06$ by Wilcoxon's test, $\mathrm{n}=5$ cells). Right,

660 comparison of change in mean EPSC $_{1}$ amplitudes plotted raw and as a box and whisker plot. $\mathrm{p}=$

6610.5 by Mann Whitney's test; $n=5$ cells per group.

662 


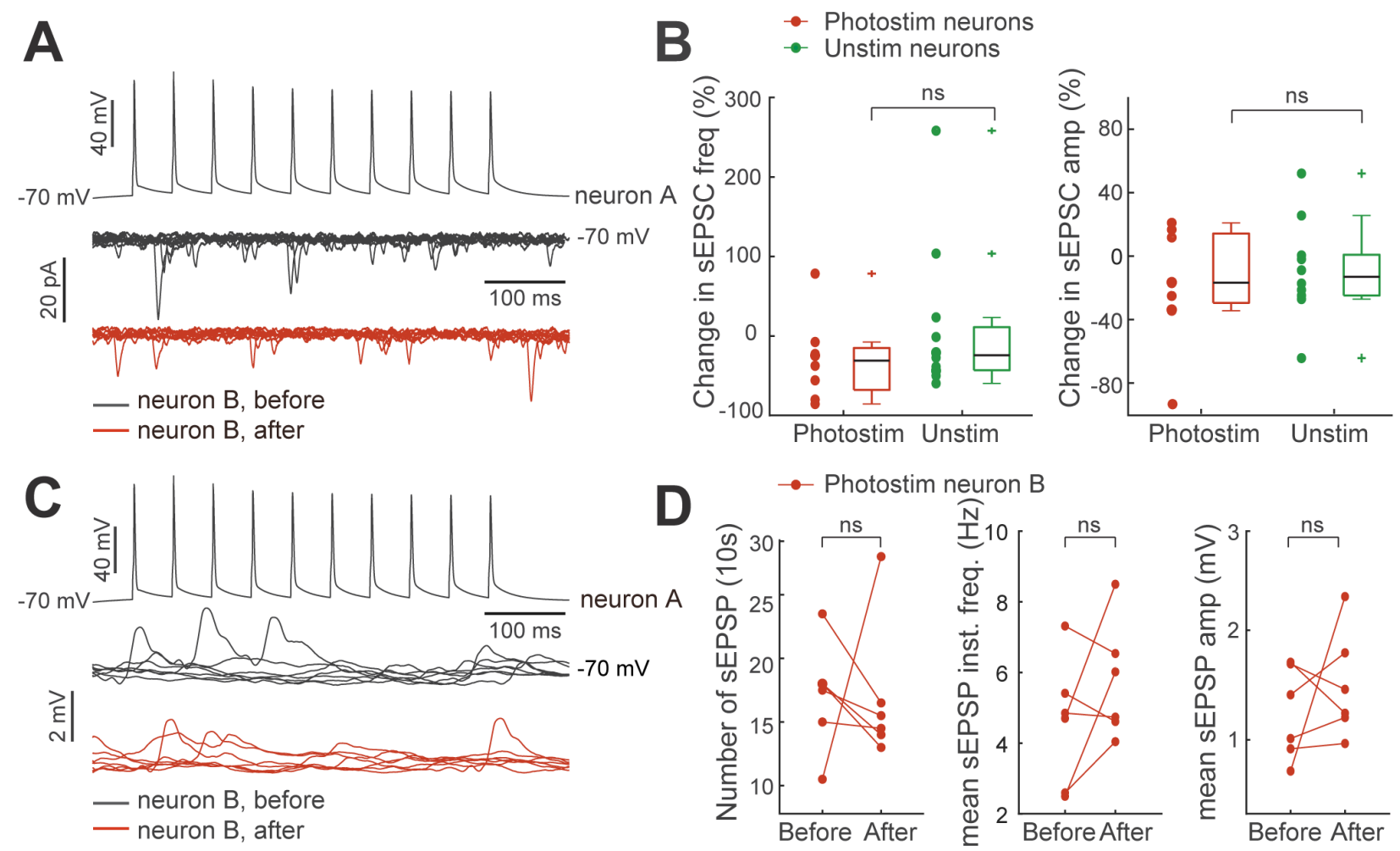

Figure 3. Lack of effect of photostimulation on spontaneous synaptic inputs

(A) Representative paired whole-cell recording of non-connected neurons. Spontaneous EPSCs remained unchanged after optogenetic photostimulation. Top: current-clamp recording of 668 presynaptic action potentials in response to $20 \mathrm{~Hz}$-train current injection (neuron A). Bottom: 669 voltage-clamp recording of 10 successive traces of spontaneous EPSCs without presynaptic 670 spike's correspondence (neuron B), before (black) and after (red) 40 min of photostimulation 671 protocol. (B) Spontaneous EPSCs frequencies of unstimulated and photostimulated neurons.

672 Change correspond to: the numerical subtraction among the first minute of EPSCs frequency and 67340 min after. Photostimulated neurons: red raw and box plot; neurons without photostimulation: 674 green raw and box plot $(p=0.4$ by Mann Whitney's test; $n=8$ for unstimulated neurons and $n=$ 67512 for photostimulated). Right: same as left but for spontaneous EPSC amplitudes. $p=0.9$ by 676 Mann Whitney's test. (C) Same as A but in current-clamp perforated patch clamp recording of 677 spontaneous EPSPs. (D) No significant difference in the number of spontaneous EPSPs, mean 
bioRxiv preprint doi: https://doi.org/10.1101/2020.07.29.223966; this version posted July 29, 2020. The copyright holder for this preprint (which was not certified by peer review) is the author/funder. All rights reserved. No reuse allowed without permission.

Alejandre-García et al., p.35

678 of instantaneous frequency and mean of amplitudes, before and after 40 min of photostimulation

679 protocol. Number of spontaneous EPSPs: $p=0.4 ;$ Instantaneous frequency average: $p=0.4$;

680 Amplitudes average: $p=0.7$. Each by Wilcoxon's test; $n=6$ photostimulated cells. 
A

Photostim start

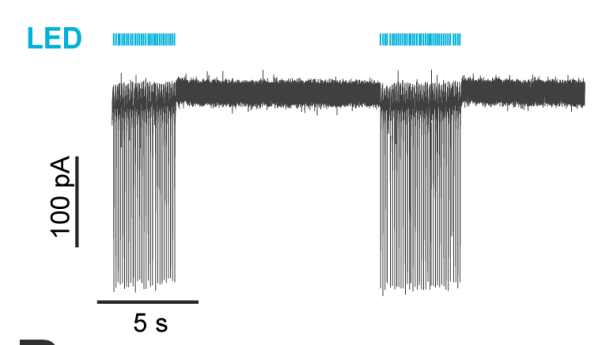

B

Before

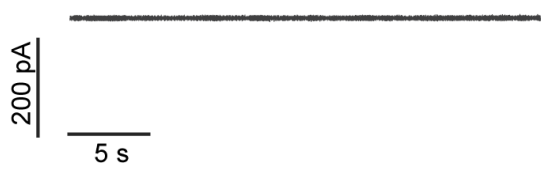

683

\section{Figure 4. Spontaneous firing rate increases after photostimulation}

(A) Neurons increase spontaneous firing after direct optogenetic photostimulation. Representative cell-attached recording of a L2/3 pyramidal neuron that expressed ST-ChroMe opsin. Left: black trace shows at the beginning of photostimulation protocol; blue lines indicated when the LED was ON. Right: red trace shows recording of the same cell after 30 min of photostimulation. (B) Spontaneous firing rate recording before (black trace) and after (red trace) 30 min of photostimulation. $60 \%$ of the cells increased their spontaneous activity (firing rate) in response to the stimulation protocol and $40 \%$ did not show changes, or instead showed a decrease in activity; $n=17$. (C) Number of spikes increased after photostimulation. Left: comparing spontaneous firing rate before and after 30 min of photostimulation (red; ${ }^{* *} \mathrm{p}<0.01$ by Wilcoxon's test; $n=17$ cells). Right: comparing spontaneous firing rate before and after 30 min without photostimulation (green; $p=0.2$ by Wilcoxon's test; $n=13$ cells). (D) Same as in $\mathbf{C}$ but for instantaneous frequency of photostimulated neurons ( ${ }^{* *} p<0.01$ by Wilcoxon's test; $n=17$ cells) and unstimulated neurons ( $p=0.3$ by Wilcoxon's test; $n=13$ cells). 

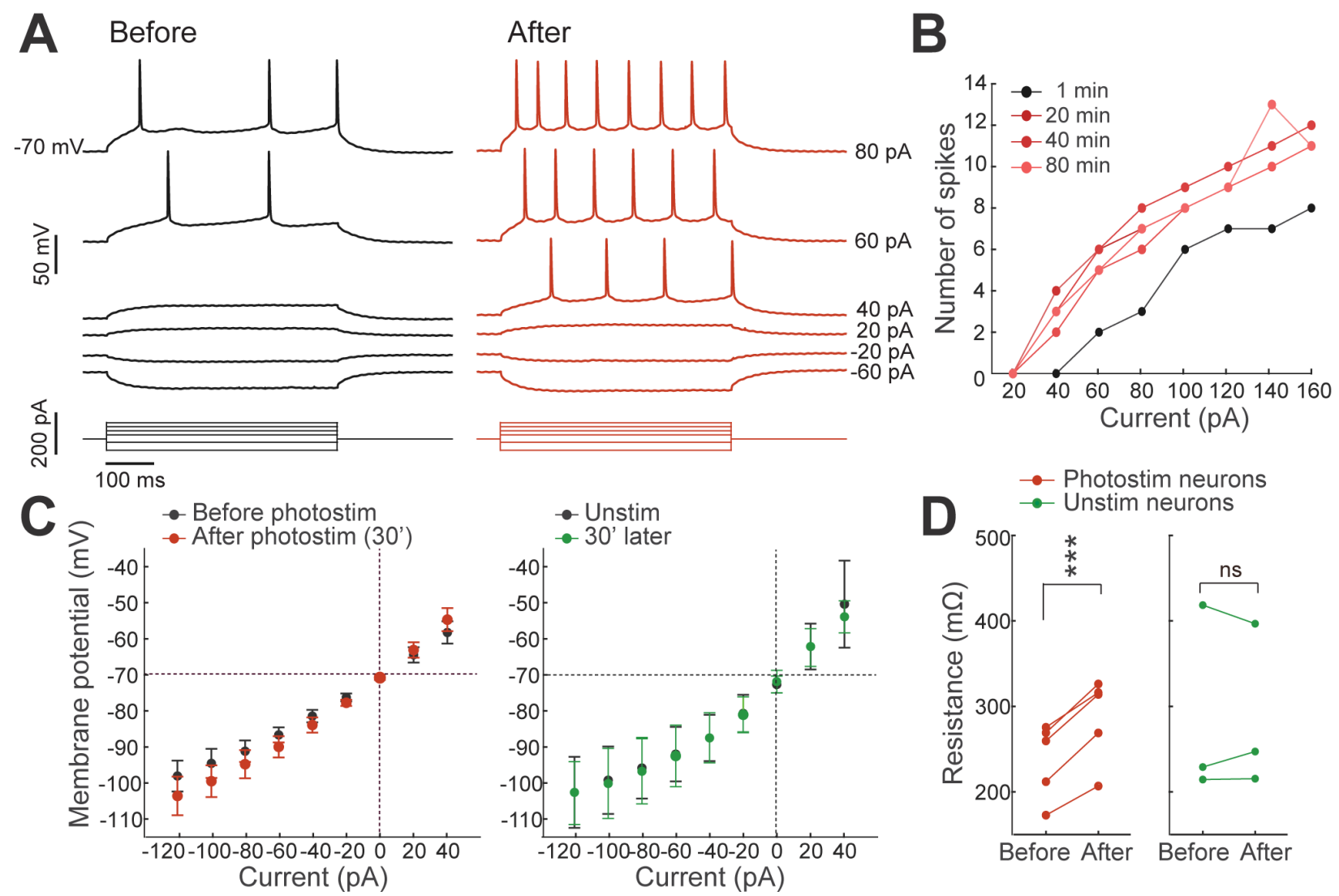

\section{Figure 5. Membrane input resistance increases after photostimulation}

(A) Membrane potential excitability increases after photostimulation. Representative membrane voltage recordings with perforated patch-clamp (top) in response $500 \mathrm{~ms}$ series of current steps (bottom). Black traces show the membrane potential changes before photostimulation and red traces after $40 \mathrm{~min}$. (B) Decrease in the rheobase after 20 min of photostimulation. Number of spikes in response to current steps of a representative photostimulated neuron, before photostimulation (at min 1): the action potentials were not evoked until $60 \mathrm{pA}$ of current injection

707 (black), after 20 min of photostimulation, neurons started to show action potentials at $40 \mathrm{pA}$ of current injection. (C) IV plot show the relation between current injection and the membrane potential (Ohm's law: Resistance $=$ V/I). IV curves showed a modification in voltage membrane when we compared before and after 30 min of photostimulation (right graph). This change was

711 not observed in the IV curves of unstimulated neurons (on the left). (D) Membrane resistance 
bioRxiv preprint doi: https://doi.org/10.1101/2020.07.29.223966; this version posted July 29, 2020. The copyright holder for this preprint (which was not certified by peer review) is the author/funder. All rights reserved. No reuse allowed without permission.

Alejandre-García et al., p.38

712 increases after photostimulation. ${ }^{* * *} \mathrm{p}<0.001$ by student's $\mathrm{t}$ test; $\mathrm{n}=5$ cells, 4 mice. Unstimulated

713 neurons remained the same at minute 1 and 30 minutes after $(p=0.9$ by student's t test; $n=3$,

7143 mice).

715 
A

\section{B}

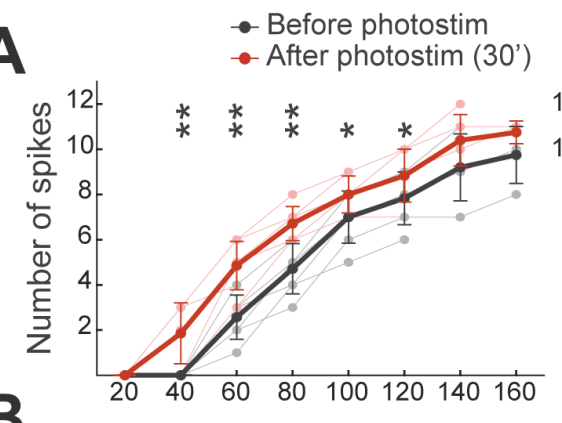

- Before photostim
- After photostim (30')
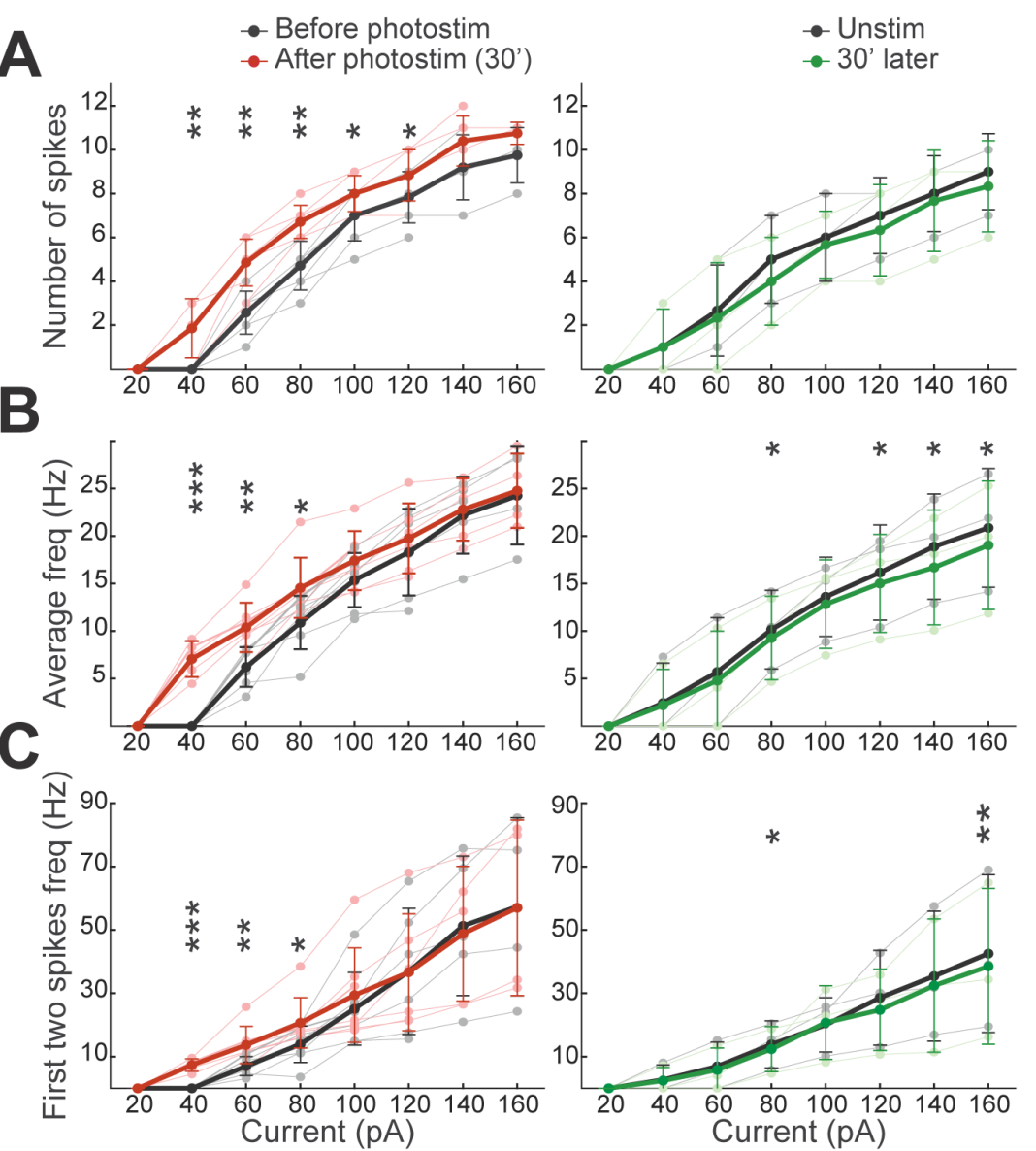

717 Figure 6. Neuronal excitability increases after photostimulation

718 (A) Neuronal activity is increased after photostimulation for the lowest current steps. The thickest

719 lines are average spikes of all neurons before (black) and after (red) photostimulation. Center:

720 average spikes of unstimulated neurons, black line corresponds to first minute and green line to

72130 minutes after. ${ }^{*} p<0.05$ and ${ }^{* *} p<0.01$ by student's t test. Right: numerical subtraction among

722 the number of spikes in the first minute and 30 minutes after, photostimulated (red) and

723 unstimulated neurons (green). ${ }^{*} p<0.05,{ }^{* *} p<0.01$ by unpaired student's $t$ test; $n=7$ for

724 photostimulated neurons and $\mathrm{n}=3$ unstimulated neurons. Data represent mean \pm SEM. (B) Mean

725 instant frequency increased for 40 to $80 \mathrm{pA}$ of current steps after photostimulation. Same as A

726 but for average of instantaneous frequency. ${ }^{*} p<0.05,{ }^{* *} p<0.01$ and ${ }^{* * *} p<0.001$ by student's $t$

727 test. Right: numerical subtraction among instantaneous frequency in the first minute and 30 min

728 after: ${ }^{*} p<0.05,{ }^{* *} p<0.01$ and ${ }^{* *} p<0.001$ by unpaired student's $t$ test. (C) Frequency of the first 
729 two action potentials increased through 40 to 80 pA of current steps after photostimulation. Same

730 as $\mathbf{A}$ but for the average of first two action potentials frequency. ${ }^{*} p<0.05,{ }^{* *} p<0.01$ and ${ }^{* * *} p<$

7310.001 by student's $t$ test. Right: Numerical subtraction among first two action potentials frequency

732 in the first minute and 30 min after: ${ }^{*} p<0.05,{ }^{* *} p<0.01$ and ${ }^{* * *} p<0.001$ by unpaired student's $t$

733 test.

734 
A
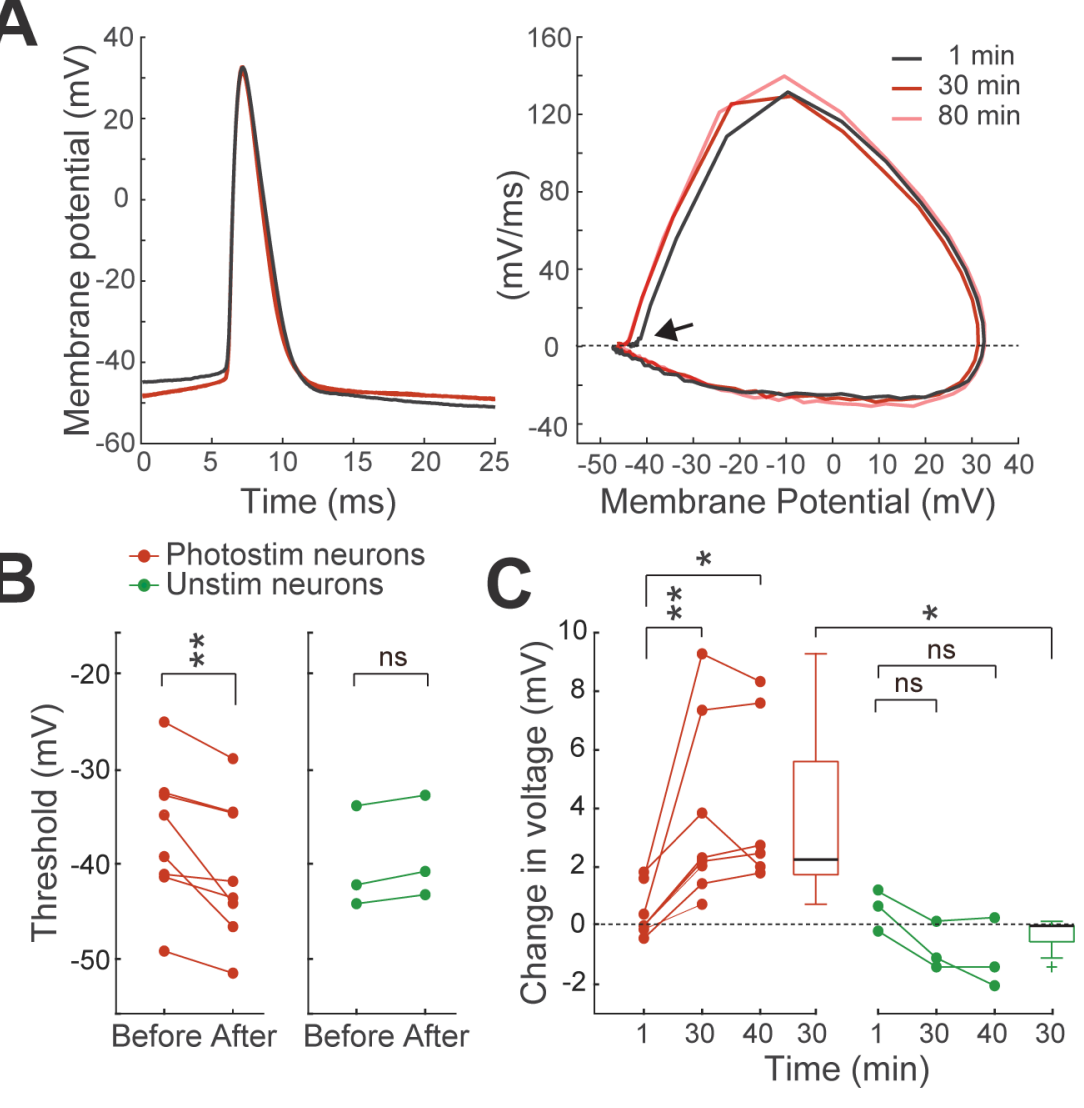

Figure 7. Spike threshold decreases after photostimulation

738 (A) Firing threshold shifts to more negative potentials after photostimulation. First action potentials

739 from recording under $60 \mathrm{pA}$ steps before (black) and after (red) 30 min of photostimulation, aligned

740 to their firing threshold. Right: Phase plot of action potentials: before start stimulation protocol

741 (black), after $30 \mathrm{~min}$ (red) and after $80 \mathrm{~min}$ (orange). Arrow shows the firing threshold position.

742 (B) Firing threshold was detected for every cell before and after photostimulation, red paired dot

743 plot ( ${ }^{* *} p<0.01$ by Wilcoxon's test; $n=8$ cells). Right: unstimulated neurons, green paired dot plot

$744(p=0.3$ by Wilcoxon's test; $n=3$ cells). (C) Voltage threshold change correspond to: numerical

745 subtraction among two values firing threshold taken in the first min before photostimulation; then,

746 numerical subtraction among first minute firing threshold and $30 \mathrm{~min}$ and 40 min later, red data

$747 \quad\left({ }^{*} p=0.05\right.$ and ${ }^{* *} p=0.01$ by Wilcoxon's test; $n=8$ cells $)$. Likewise, for unstimulated neurons,

748 green data ( $p=0.25$ by Wilcoxon's test; $n=3$ cells). Photostimulated neurons: red box plot and 
bioRxiv preprint doi: https://doi.org/10.1101/2020.07.29.223966; this version posted July 29, 2020. The copyright holder for this preprint (which was not certified by peer review) is the author/funder. All rights reserved. No reuse allowed without permission.

Alejandre-García et al., p.42

749 for unstimulated neurons: green box plot. ( ${ }^{*} p<0.05$ by Mann Whitney's test; $n=8$ for 750 photostimulated and $\mathrm{n}=3$ for unstimulated cells). 
A

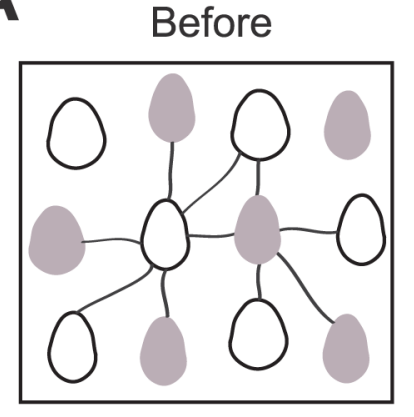

B

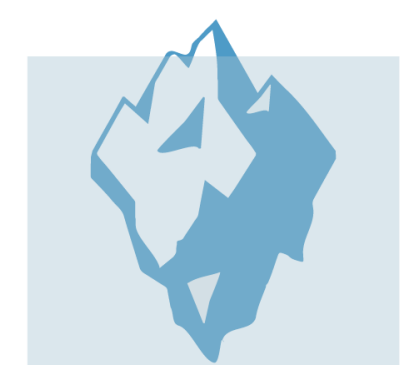

After
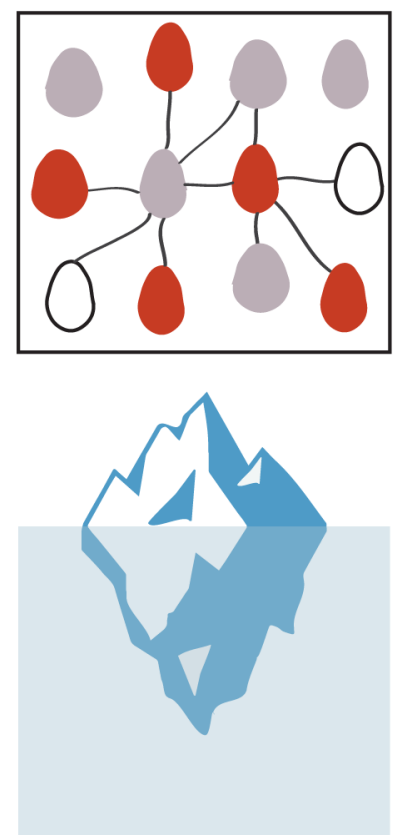

Input response:

- Firing action potentials

EPSPs

No response

Figure 8. Iceberg emergence model of ensemble formation.

755 (A) Emergence of ensembles after increases in neuronal excitability. Illustration of a diversity in responses to an input of a neuronal population, before and after coactivation (or photostimulation

757 in the case of this study). Color corresponds to membrane potential in response to an input: white

758 are resting membrane potentials, gray are subthreshold responses and red are suprathreshold

759 ones, with firing of action potentials. After photostimulation neurons are more excitable, so the

760 same inputs induce some of them to fire (red cells).

761 (B) Left: Iceberg emergence model: An iceberg with higher density keeps mostly underwater.

762 (Right) But if its density decreases, the iceberg emerges above water. Density is an intrinsic

763 property, and, by changing it, the iceberg changes its response to the same environment.

764 Likewise, for a neuronal ensemble, membrane resistance and firing threshold are neuronal

765 intrinsic property that are being modified, and they enhance its response to the same excitatory

766 input intensity, resulting in an increase depolarization and action potentials. 